\title{
Signal Degradation due to Charge Buildup in Noble Liquid Ionization Calorimeters
}

\author{
John P. Rutherfoord* \\ Department of Physics, University of Arizona, Tucson, AZ, 85721 USA
}

\begin{abstract}
We calculate the degradation in the signal from sampling, noble liquid, ionization calorimeters due to charge build-up from the slowly drifting positive ions. Such effects can be significant in calorimeters planned for operation at high luminosity hadron colliders such as the Large Hadron Collider (LHC) at CERN.
\end{abstract}

Key words: liquid argon calorimetry

PACS: 29.40.Vj

\section{Introduction}

In the high rate environments anticipated for the next round of hadron colliders, such as the Large Hadron Collider (LHC) at CERN, electromagnetic and hadronic calorimeters for measuring particle energies will play a prominent role and face difficult challenges. One major concern is radiation damage. Sampling calorimeters using conventional scintillators will suffer gain variations and light attenuation from darkening of the scintillator by massive radiation doses. On the other hand, noble liquid calorimeters can readily be made "rad hard" if the readout preamps are located remotely. The noble liquid, absorber plates, charge collection pads, and cables can all be selected to withstand the highest predicted doses. While the full response from an ionization calorimeter is not as fast as some other technologies, the signal can be shaped to times of order the bunch crossing interval. For this reason it is thought that a noble liquid calorimeter is an attractive option at luminosities of $10^{34} \mathrm{~cm}^{-2} \mathrm{sec}^{-1}$ including calorimetry in the particularly difficult forward regions.

\footnotetext{
* Corresponding author.

Email address: rutherfo@physics.arizona.edu (John P. Rutherfoord).
} 


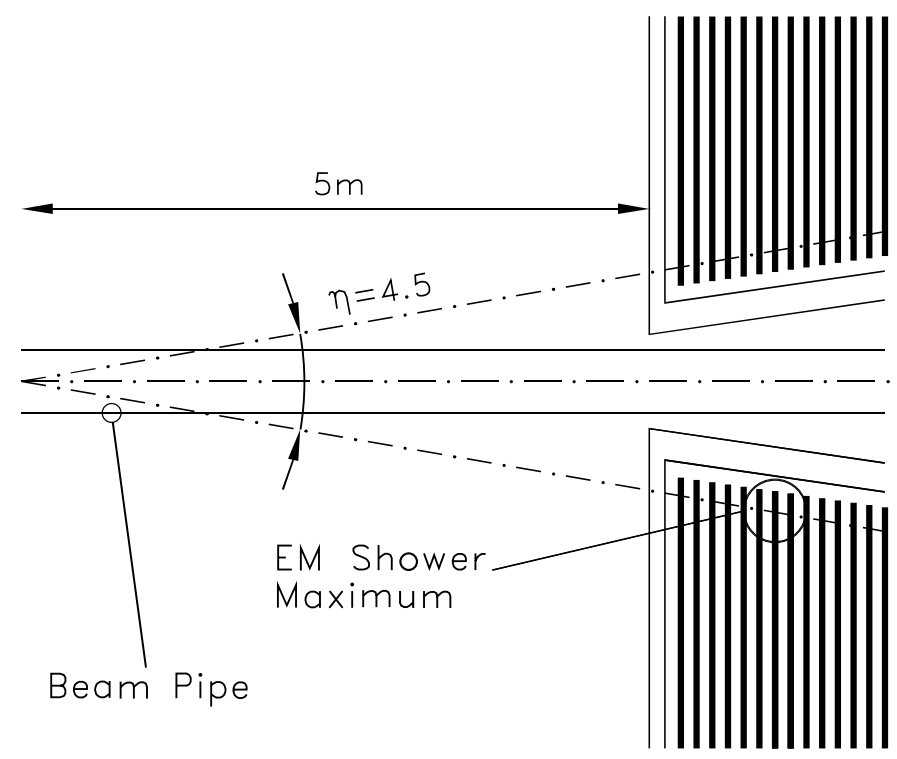

Fig. 1. Schematic diagram of a parallel plate, liquid argon forward calorimeter. Also shown are the cryostat walls and the region of EM shower maximum.

While the electrons liberated from argon, krypton, or xenon atoms by passage of high energy charged particles through the liquid gap drift quickly in the electric field, the positive ions move much more slowly. In the high rate environment of the LHC, low energy particles are constantly showering in the calorimeter. In a given volume of liquid, particularly near electromagnetic shower maximum, the rate of ionization is large enough that positive ions accumulate in the gap and distort the electric field. When a high energy particle hits the calorimeter the large shower liberates excess electrons (and ions) on top of the background production. The distorted electric field degrades the signal from these drifting electrons. In this paper we calculate the effect of the build-up of positive ions on the electric field and on the signal in a single gap of a sampling, noble liquid ionization calorimeter.

Fig. 1 is a schematic of a forward calorimeter with jet coverage over the range $3<|\eta|<4.5$. The calorimeter extends to values of $|\eta|$ above 4.5 in order to contain the EM portion of hadronic jets at $|\eta|=4.5$.

For specific rate calculations we will consider a liquid argon sampling gap located near electromagnetic shower maximum as indicated in the circle in Fig. 1. We will find that without proper design, liquid ionizing calorimeters will fail to operate adequately not only at $|\eta|=4.5$ but at significantly lower values of $|\eta|$ as well. 


\section{Principle of an Ionization Calorimeter}

At atmospheric pressure argon liquefies at $87.3 \mathrm{~K}$ and solidifies at $83.9 \mathrm{~K}$. Argon is a nonpolar, dielectric liquid, i.e. it is an excellent insulator with a large breakdown potential. However when free electrons are introduced they readily drift opposite the direction of an applied electric field with little loss, i.e. the electrons do not attach to argon atoms. High energy charged particles, such as cosmic ray muons, ionize the argon atoms producing free electrons. The minimum energy loss for light particles (minimum ionization) is about $d E / d x=1.51 \mathrm{MeV} / \mathrm{g} / \mathrm{cm}^{2}$. Using a density of $\rho=1.40 \mathrm{~g} / \mathrm{cm}^{3}$ gives $d E / d x=$ $2.11 \mathrm{MeV} / \mathrm{cm}$. For every $\sim 25 \mathrm{eV}$ of energy lost in the argon by such a high energy charged particle, one ion pair is created so a minimum ionizing particle traversing the gap produces about 8500 ion pairs $/ \mathrm{mm}=1.4 \mathrm{fC} / \mathrm{mm}$ of each charge along its track. These ion pairs recombine unless an electric field is present. For electric fields of order $1 \mathrm{kV} / \mathrm{mm}$ the electrons drift quickly $(\sim 5$ $\mathrm{mm} / \mu \mathrm{sec}$ at $\mathrm{T} \sim 85 \mathrm{~K}$ ) leading to a measurable current whose initial value is $6.8 \mathrm{nA}$ for a minimum ionizing particle at normal incidence. The positive charges drift far more slowly and the consequences are the focus of this paper.

In 1974 Willis and Radeka [1] introduced the liquid argon sampling calorimeter for use in high energy physics. A high energy particle incident on such a calorimeter creates more particles of lower energy, primarily in interactions in the heavy metal plates between the sampling gaps. These particles, in turn, produce more particles of even lower energy. Repeated interactions produce a shower of particles which is quenched when the energies of the particles fall below various thresholds, e.g. for particle production. The number of particles produced in the shower is nearly proportional to the energy of the incident particle. And because the number of ionization electrons is proportional to the number of charged shower particles, the electron current is a measure of the incident particle energy.

Liquid argon is an ideal choice for the sensitive medium in a sampling calorimeter because it is relatively inexpensive (the cost of the gas from the producer at the requisite purity, per liquid volume, is roughly the cost of milk), the number of ion pairs produced per track length of high energy charged particles is large, and the ionization electrons drift freely and quickly in the liquid argon. While not inexpensive, krypton and xenon otherwise have quite similar properties. However when numerical values are required we will choose argon as example. 


\section{Plan of Attack}

The mathematics required for a complete description of the behavior of drifting electrons and ions in a liquid argon gap is complicated and can obscure many of the dominant aspects of the problem. For this reason we employ a number of simplifying assumptions and approaches, motivating them when they are introduced. This allows us to develop analytic expressions which are approximately correct over a range of parameter values. We can then explore a parameter space in order to optimize a design and minimize the ion loading problem. But care must be exercised in using the expressions outside their range of validity.

In the next section we review the measurements of the positive ion mobility in argon and place these measurements in context. Section 5 introduces the issue of recombination which is taken up later in this paper. We use section 6 to establish the approach and to develop some intuition for more difficult situations to be encountered later. In section 7 the steady-state configurations of charge, current, and electric field in the gap are calculated at first order for various values of the ionization rate and section 8 provides some necessary higher order corrections. The way in which the steady-state ionization affects a transient signal event is calculated in section 9 and simulation results are presented in section 10. In section 11 we estimate the fluctuations in what we have assumed is a steady-state ionization rate. Finally in section 12 we translate the calculations to a practical situation and estimate the limits of operation of liquid argon calorimeters.

\section{Electron and Ion Drift Velocities}

The drift velocity of free electrons in liquid argon is well measured [2-8] and there is some understanding of the results $[9,10]$. At very low electric fields the electron drift velocity is proportional to the applied electric field and so is described by an electron mobility $\mu_{-} \equiv \mathrm{v}_{-} / E$. At modest electric fields the drift velocity saturates, that is, it appears to approach a constant value independent of electric field. For purposes of analytic calculation in this paper we will assume that the electron drift velocity, $\mathrm{v}_{-}=\mathrm{v}_{-}(E)$, depends on electric field, $E$, as shown in Fig. 2. (Note that throughout this paper the symbol v refers to the drift velocity as a function of electric field while the symbol $v$ refers to the drift velocity at the specific value of electric field $E=V_{o} / a$ where $V_{o}$ is the nominal potential applied across the gap, considered a constant, and $a$ is the gap width (see Fig. 3), i.e.

$$
v_{-}=\mathrm{v}_{-}\left(V_{o} / a\right) .
$$




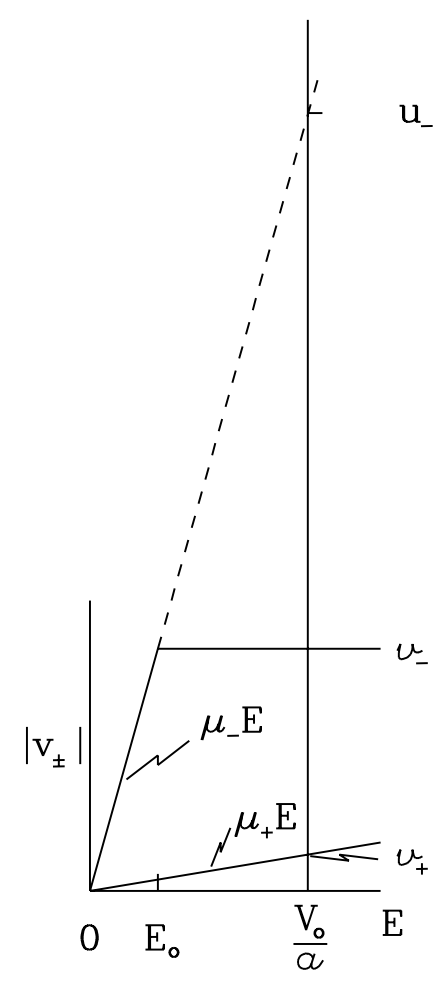

Fig. 2. Electron (-) and positive ion (+) drift velocities (not to scale) as a function of electric field as used in the analytic calculations. The electron velocity at low fields is described by a mobility which, when extrapolated to the nominal electric field of the gap, gives a velocity of $u_{-}$.

Furthermore we always take $\mathrm{v}_{-}, v_{-}$, and $\mu_{-}$as positive and insert explicit negative signs in the equations to indicate direction opposite the electric field direction.) We assume that below some value of electric field $E_{o}$ (which we will take to be very small relative to $V_{o} / a$ ) the drift velocity $\mathrm{v}_{-}=\mu_{-} E$ while for $E>E_{o}$ we assume $\mathrm{v}_{-}=v_{-}$, i.e. $\mathrm{v}_{-}$is independent of electric field $\left(v_{-} \sim 5\right.$ $\mathrm{mm} / \mu \mathrm{sec})$. Of course $\mu_{-} E_{o}=v_{-}$in order for $\mathrm{v}_{-}$to be a continuous function of E.

Less well understood is how positive charge leaves the gap. We will assume in liquid argon (as in argon gas) that positive argon ions drift in the electric field and that $\mathrm{v}_{+}=\mu_{+} E$ where $\mu_{+}$is independent of $E$. This is also shown in Fig. 2. Using our convention for $\mathrm{v}$ and $v$, we note that $v_{+}=\mu_{+} V_{o} / a$.

Assuming drifting positive argon ions are the dominant positive charge carriers in liquid argon, it is not known whether the ions are monatomic or not. The $\mathrm{Ar}^{+}$ions could form ionized molecules such as $\mathrm{Ar}_{2}^{+}$or $\mathrm{Ar}_{3}^{+}$. So the mass of the positive charge carrier is unknown. In solid argon it is not clear whether there is a transport of positive charge $[11,12]$. If there is, then it is likely due to hole 


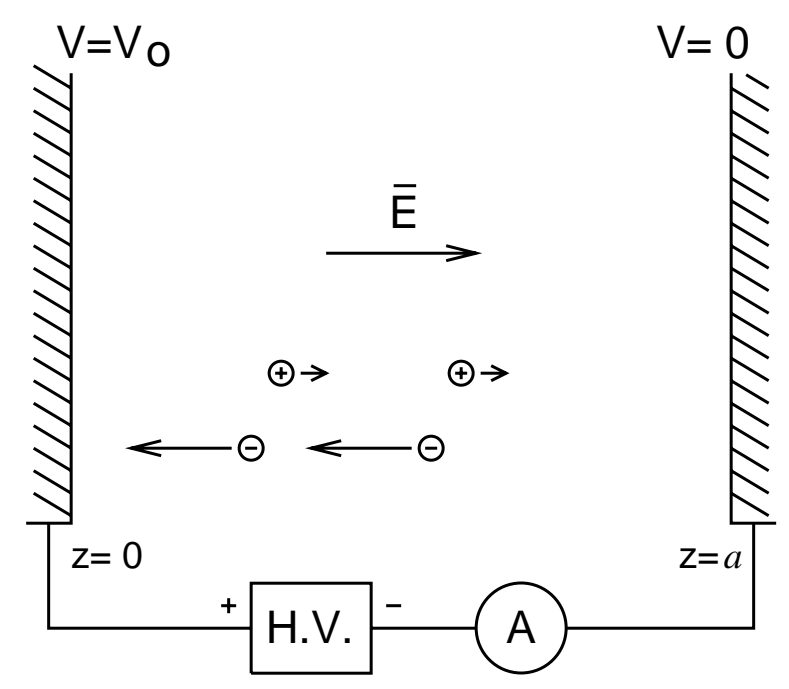

Fig. 3. A schematic diagram of a liquid argon gap. The plate at $z=0$ is at a positive high voltage $V_{o}$ while the plate at $z=a$ is at ground. Electrons drift to the left and positive ions to the right. The current is measured by device A. It is assumed that the circuit connecting the plates has zero impedance.

transport rather than the motion of positive ions. So it is possible that hole transport dominates in liquid argon also. Or it is possible that no mechanism dominates. Positive charge may move through liquid argon due to drifting $\mathrm{Ar}^{+}, \mathrm{Ar}_{2}^{+}, \ldots$ and hole transport. This possibility may help explain why the experimental measurements, which we review below, are so disparate.

Table I summarizes the measured positive ion mobilities in liquid argon [1319]. The lowest value is $\mu_{+}=0.02 \mathrm{~mm}^{2} / \mathrm{Vs}$ while the highest is about $\mu_{+}=1$ $\mathrm{mm}^{2} / \mathrm{Vs}$, a factor of 50 variation from lowest to highest. It should be pointed out that the lowest value of $\mu_{+}$was not actually measured but was extrapolated from larger values [13]. The experimenters believed they saw evidence that drifting positive ions set up a bulk fluid flow which carried the positive ions along faster. Lower densities of positive ions gave lower drift velocities at the same field. If this is true then the measurement should also be geometry dependent.

The highest values of $\mu_{+}$in Table I were determined indirectly via phenomenological models of the experimental data $[18,19]$. In these cells ions were produced by electric field ionization at the tip of a sharp electrode. It is possible that liquid argon in the vicinity of the tip was vaporized making the result somewhat uncertain. Since the positive ion mobility measurement was not the primary focus of these experiments the results can be considered a by-product and perhaps given less weight. 
Table I

Positive Ion Mobilities in Liquid Argon

\begin{tabular}{|c|c|c|c|c|}
\hline Reference & Source & Cell & $\begin{array}{c}\text { Field } \\
(\mathrm{kV} / \mathrm{mm})\end{array}$ & $\begin{array}{c}\mu_{+} \\
\left(\mathrm{mm}^{2} / \mathrm{Vs}\right)\end{array}$ \\
\hline Dey, Lewis '63 & $\mathrm{Am}^{241}$ & Plates /w grid & Up to 1 & 0.02 \\
\hline Davis, Rice, Meyer '62 & $\mathrm{Po}^{210}$ & Plates /w grid & $\leq 0.025$ & 0.06 \\
\hline Henson '64 & Field Ion & Shutter/Drift/TOF & $0-0.43$ & $0.06-0.0975$ \\
\hline Gee et al. ' 85 & X-rays & Plates & $0.06-2.7$ & 0.2 \\
\hline Williams '57 & $\mathrm{Po}^{210}$ & Plates & $2.4-18.7$ & 0.28 \\
\hline Halpern, Gomer '69 & Field Ion & Tip/Plate & $\sim 0.1$ & $0.7-1.0$ \\
\hline Arii, Schmidt '84 & Field Ion & Tip/Plate & & $0.74-1.16$ \\
\hline
\end{tabular}

The range of electric fields employed for each measurement is tabulated when given by the authors. No particular electric field dependence is apparent. Some experiments varied the temperature and pressure as well and saw no striking dependence over the narrow range near the argon boiling temperature at one atmosphere.

We conclude that little is known about the positive charge transport mechanism in liquid argon and that the summary parameter of relevance to us, the positive ion mobility, is highly uncertain. We will continue to assume that some constant value for the positive ion mobility exists, just that it is not presently well known, but we keep in mind the possibility that a description of the motion of positive charge in liquid argon is not adequately represented by a single constant value of $\mu_{+}$.

Table II puts the positive ion drift velocity into perspective by presenting a number of other relevant velocities. Note that the thermal velocities are much larger than the drift velocities for the electric fields of relevance here. Also note that thermal is in quotes for electrons because the free electrons in an electric field become "super heated" due to the lack of an efficient energy loss mechanism below about $1 \mathrm{eV}[9,10]$. The table also justifies our neglect of diffusion velocity in subsequent calculations [20]. In estimating a diffusion velocity we've made the extreme assumption that the number density, $n$, of positive ions varies by $100 \%$ over a distance of $0.001 \times a$ where $a$ is the gap width of $2 \mathrm{~mm}$. For the diffusion constant $D$ we took a value corresponding to the largest measured value of $\mu_{+}$. 
Table II

Velocities in Liquid Argon

Electron drift velocity at $E=1 \mathrm{kV} / \mathrm{mm}$

$v_{-}=5 \times 10^{0} \mathrm{~mm} / \mu \mathrm{s}$

Low frequency sound

$c=0.85 \times 10^{0} \mathrm{~mm} / \mu \mathrm{s}$

RMS "thermal" velocity of electron at $1 \mathrm{kV} / \mathrm{mm}$

$\sqrt{\left\langle v_{-}^{2}\right\rangle}=8 \times 10^{2} \mathrm{~mm} / \mu \mathrm{s}$

Positive ion drift velocity at $E=1 \mathrm{kV} / \mathrm{mm}$

$$
\begin{array}{crl}
\text { assuming } \mu_{+}=0.02 \mathrm{~mm}^{2} / \mathrm{Vs} & v_{+} & =2 \times 10^{-5} \mathrm{~mm} / \mu \mathrm{s} \\
\text { assuming } \mu_{+}=1 \mathrm{~mm}^{2} / \mathrm{Vs} & v_{+} & =1 \times 10^{-3} \mathrm{~mm} / \mu \mathrm{s} \\
\text { RMS thermal velocity of } \mathrm{Ar}^{+} \text {ion at } \mathrm{T}=90 \mathrm{~K} & \sqrt{<v_{+}^{2}>} & \sim 2.4 \times 10^{-1} \mathrm{~mm} / \mu \mathrm{s}
\end{array}
$$

Diffusion velocity $\left\langle v_{z}\right\rangle=-D \frac{1}{n} \frac{d n}{d z}$

$$
\text { for } \frac{d n}{n}=1 \text { and } d z \sim a / 1000 \quad<v_{z}>\sim 4 \times 10^{-6} \mathrm{~mm} / \mu \mathrm{s}
$$

\section{Recombination}

At high ionization rates recombination figures prominently. In the literature three types of recombination are identified. Here we discuss each in turn.

\subsection{Bulk Recombination}

Bulk recombination occurs when there is a continuum of charges of both types present at the same time. A random electron combines with a random positive ion at a rate per unit volume proportional to the product of the electron and ion densities, i.e.

$$
D_{r}=R_{r} n_{+} n_{-}
$$

where $D_{r}$ is the recombination rate per unit volume, $R_{r}$ is the rate constant, and $n_{+}$and $n_{-}$are the number densities of electron and ion charges. Bulk recombination will be important in this paper.

\subsection{Columnar Recombination}

Columnar recombination occurs when a high energy charged particle, parallel to the applied electric field, produces a column of ion pairs which, as the 
electrons drift in one direction and the positive ions in the other, pass each other and occasionally recombine. This is important when the ionizing particle tracks are parallel to the electric field. However this form of recombination will not be relevant to us because 1) the ionizing particle tracks are divergent near EM shower max due to multiple scatter and 2) the electrode structures (e.g. accordion and tube) in modern liquid argon calorimeters have electric fields at odd angles to particles from the interaction point.

\subsection{Initial, Germinate, or Geminate Recombination}

Initial recombination (sometimes also called geminate or germinate recombination) refers to the process where an electron, freed from an argon atom and thus producing a positive argon ion, returns to that same ion and recombines to produce an argon atom again. With no external electric field it is presumed that this mechanism leads to the recombination of all pairs. At sufficiently high external fields and at low ionization density, all pairs are permanently separated. Between these extremes pairs are more likely to remain free as the electric field increases. We will parameterize the probability for a pair to remain free as [20]

$$
P(E)=\left(E / E_{2}\right) \ln \left(1+E_{2} / E\right)
$$

where $E$ is the electric field. For analytic calculations we choose $E_{2} \ll V_{o} / a$ so initial recombination is neglected. For simulations $E_{2}$ is chosen to approximate data, $E_{2} \sim 84 \mathrm{~V} / \mathrm{mm}$.

The value of $E_{2}$ is sensitive to certain contaminants in the liquid argon. Sufficiently well designed experiments are able to separately measure the effects of contaminants which induce attachment, such as oxygen, even if the contaminant cannot be eliminated. Other contaminants are conjectured to catalyze initial recombination so their effects cannot be separately determined unless the contaminant can be completely removed or the impurity concentration accurately controlled allowing extrapolation to zero concentration.

\section{$6 \quad$ Linear Model and Some Examples}

When the gap width $a$ is small compared to the transverse dimensions, assuming that the plates are infinitely large is a good approximation. We will develop a one-dimensional model where the parameters of interest depend only on $z$, the distance across the gap, and vary so slowly with $x$ and $y$ as to be nearly constant. The one dimensional model assumes that charges are deposited in 
the gap in planes parallel to the plates of uniform surface charge density $\sigma$. Here uniform means independent of $x$ and $y$. This is equivalent, in some sense, to an electrostatics problem defined along a line, e.g. along the $z$ axis in our case.

For a single ionizing particle traversing a gap at a small angle to the $z$ axis this is a poor approximation. But for multiple electromagnetic showers near shower maximum with Moliere radius of several centimeters the ionization density doesn't change in $x$ and $y$ so rapidly on the scale of the gap width $a$ of $2 \mathrm{~mm}$. So a linearized model, while not an excellent description, will be a reasonable model for our purposes.

Fig. 3 shows the geometry of the gap we will consider throughout this paper. The electric field is aligned along the positive $z$ direction. So the electrode at $z=0$ is at potential $V_{o}$ and the electrode at $z=a$ is at ground. A very schematic readout is indicated by an ammeter. We will assume a zero impedance readout so that the potential across the gap is always at $V_{o}$ regardless of the current drawn.

The surface charge density on the plate at $z=0$ is $\sigma(0)=\epsilon E(0)$ and on the plate at $z=a, \sigma(a)=-\epsilon E(a)$. With no charges in the gap, $E(0)=E(a)=$ $V_{o} / a$ and $\sigma(a)=-\sigma(0)$ so the plate at $z=0$ is positively charged and the plate at $z=a$ is negatively charged.

The following examples help establish our method and provide some intuition for the case of interest considered in section 9. In each we assume that ionization is deposited in the gap at the instant $t=0$.

\subsection{Example 1}

Suppose we introduce in the gap a sheet of surface charge density $\sigma_{+}\left(\ll \epsilon V_{o} / a\right)$ at $z=z_{+}$. Gauss' Law for this geometry gives

$$
\epsilon \frac{\partial E}{\partial z}=\rho(z)
$$

and setting $\rho(z)=\sigma_{+} \delta\left(z-z_{+}\right)$we get

$$
E=E_{g}+\theta\left(z-z_{+}\right) \sigma_{+} / \epsilon
$$


where $\theta$ is the unit step function and where $E_{g}$ is chosen so that $\int_{0}^{a} E \mathrm{~d} z=V_{o}$. We find $a E_{g}+\left(a-z_{+}\right) \sigma_{+} / \epsilon=V_{o}$ or

$$
E= \begin{cases}V_{o} / a-\left(1-z_{+} / a\right) \sigma_{+} / \epsilon & 0<z<z_{+} \\ V_{o} / a+\left(z_{+} / a\right) \sigma_{+} / \epsilon & z_{+}<z<a\end{cases}
$$

Now suppose the charge sheet $\sigma_{+}$moves to the right with velocity $v_{+}$, then the charge density on the plates changes as

$$
\frac{d \sigma(0)}{d t}=-\frac{d \sigma(a)}{d t}=\epsilon \frac{d E(0)}{d t}=\epsilon \frac{d E(0)}{d z_{+}} \frac{d z_{+}}{d t}=\epsilon \frac{d E(0)}{d z_{+}} v_{+}=\frac{v_{+} \sigma_{+}}{a}
$$

The changing surface charge density on the plates requires that a current flow through the external circuit. Per unit area of the plates, this current is given by

$$
J=v_{+} \sigma_{+} / a
$$

which is equivalent to the average current density of the moving charge sheet within the gap. That is, a current is induced in the external circuit by the moving charges within the gap. If $1 \mathrm{pC} / \mathrm{mm}^{2}$ moves through the gap from the plate at $z=0$ to the plate at $z=a$, then $1 \mathrm{pC} / \mathrm{mm}^{2}$ of electrical charge will flow from one plate to the other through the external circuit as well.

Fig. 4a shows the electric field within the gap as a function of $\mathrm{z}$. Fig. 4b shows $E(a)$ and $E(0)$, the electric field at the surface of the two plates, as a function of $z_{+}$, the location of the charge sheet. Fig. 4c shows $E(a)$ and $E(0)$ as a function of time assuming the charge sheet is initially placed at $z_{+}=0$ and then drifts at uniform velocity $v_{+}$towards the plate at $z=a$. Fig. $4 \mathrm{~d}$ shows the induced current due to this drifting charge. Note that when the charge sheet hits the plate at $z=a$, i.e. when $z_{+}=a$, then $E(a)$ changes abruptly so that the induced current exhibits a negative delta function of area $\sigma_{+}$. This is exactly balanced by the charge current due to the charge sheet reaching the plate at $z=a$ so that the total current, induced plus charge, has no negative delta function.

\subsection{Example 2}

Suppose next that between the same two plates we introduce in the gap a sheet of positive surface charge density of positive ions $\sigma_{+}=\sigma_{o}$ at $z=z_{+}$and a sheet of negative charge density of electrons $\sigma_{-}=-\sigma_{+}=-\sigma_{o}$ also at $z=z_{+}$.

In this example the electric fields at the plates are $V_{o} / a$ as they were with no 

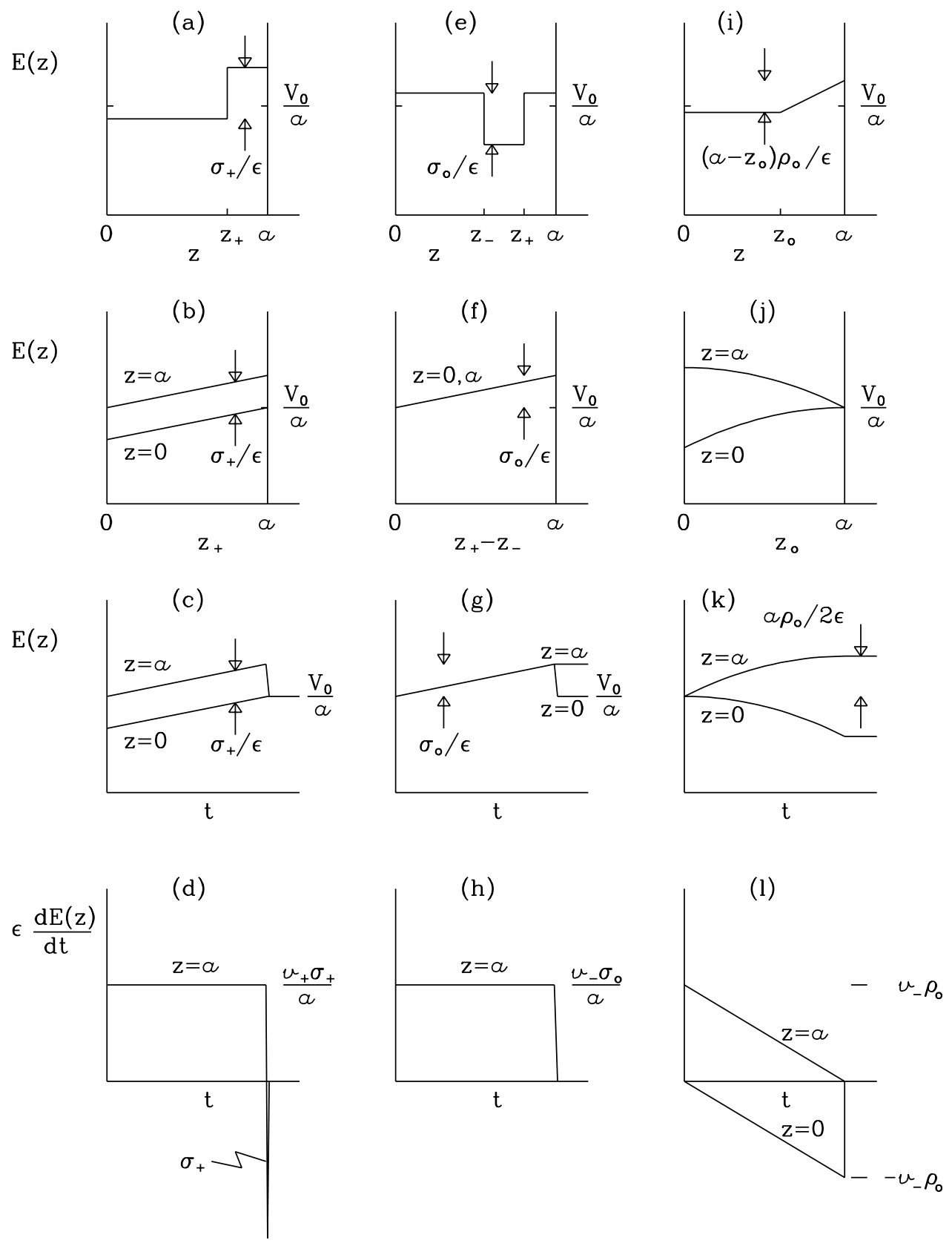

Fig. 4. Electric field and change of electric field with time for examples 1 to 3 in the text.

charges in the gap. This is a somewhat more realistic example corresponding, in the 3-D case, to an ion pair created within the gap by, say, photo-ionization.

For simplicity, and as a realistic approximation, suppose that the drift velocity of the electrons is $v_{-}$and that the positive ions are immobile. Then at some 
later time the electron charge sheet will have drifted to the left to position $z=z_{-}$and the positive ion charge sheet will remain at $z=z_{+}$. It is easy to show that the electric field in the gap (always requiring $\int_{0}^{a} \mathrm{~d} z E=V_{o}$ ) will be

$$
E= \begin{cases}V_{o} / a+\left(z_{+} / a-z_{-} / a\right) \sigma_{o} / \epsilon & 0<z<z_{-} \\ V_{o} / a+\left(z_{+} / a-z_{-} / a-1\right) \sigma_{o} / \epsilon & z_{-}<z<z_{+} \\ V_{o} / a+\left(z_{+} / a-z_{-} / a\right) \sigma_{o} / \epsilon & z_{+}<z<a\end{cases}
$$

and that the change in the surface charge density at each of the plates is

$$
\frac{d \sigma(0)}{d t}=-\frac{d \sigma(a)}{d t}=\epsilon \frac{d E(0)}{d t}=\sigma_{o} v_{-} / a
$$

and so the induced current per unit area of plate in the external circuit is again $J=\sigma_{o} v_{-} / a$.

Fig. 4e shows the electric field within the gap as a function of $z$. Fig. 4f shows $E(a)$ and $E(0)$, the electric field at the surface of the two plates, as a function of $z_{+}-z_{-}$, the distance between the separating charge sheets. Fig. $4 \mathrm{~g}$ shows $E(a)$ and $E(0)$ as a function of time assuming that $z_{+}=a$, i.e. that the two charge sheets started at $z=a$ (or just a small amount less than $z=a$ ). Fig. $4 \mathrm{~h}$ shows the induced current due to the separating charge sheets.

Generalizing this example to the case where the positive and negative surface charge densities aren't necessarily equal and both charge carriers can drift we will find

$$
J=\sigma_{+} v_{+} / a+\left|\sigma_{-}\right| v_{-} / a
$$

When $\sigma_{+}=-\sigma_{-}$and $v_{+}=v_{-}$, twice the current will be observed in the external circuit compared to the case above with immobile positive ions. But usually $v_{+} \ll v_{-}$.

\subsection{Example 3}

Now assume the whole gap is uniformly filled with immobile positive ions of charge density $\rho_{+}=\rho_{o}\left(\rho_{o} \ll V_{o} \epsilon / a^{2}\right)$ and mobile electrons of charge density $\rho_{-}=-\rho_{o}$ such that the net charge density within the gap is zero and the electric field is $V_{o} / a$ everywhere as it was with no charge in the gap at all. This case is the linearized analog of the ionization deposited by a high energy charged particle traversing the gap in the $\mathrm{z}$ direction leaving uniform ionization. And, as we argued earlier, it is an approximation to an electromagnetic shower near shower maximum. 
After some time the electrons will drift to the left (and some will collect on the plate at $z=0$ ) leaving a region of the gap from $z_{o}$ to $a$ free of electrons. (We're neglecting diffusion.) Using Gauss' Law we find the electric fields in the gap at this time are

$$
E=\left\{\begin{array}{lr}
V_{o} / a-\left(1-z_{o} / a\right)^{2} \rho_{o} a / 2 \epsilon & 0<z<z_{o} \\
V_{o} / a-\left(1-z_{o} / a\right)^{2} \rho_{o} a / 2 \epsilon+\left(z-z_{o}\right) \rho_{o} / \epsilon & z_{o}<z<a
\end{array}\right.
$$

In this case we find at the plates

$$
\begin{gathered}
\epsilon \frac{d E(0)}{d t}=\frac{d \sigma(0)}{d t}=-\rho_{o} v_{-}\left(1-z_{o} / a\right)=-\rho_{o} v_{-}^{2} t / a \\
\epsilon \frac{d E(a)}{d t}=\frac{-d \sigma(a)}{d t}=+\rho_{o} v_{-}\left(z_{o} / a\right)=\left(\rho_{o} v_{-} / a\right)\left(a-v_{-} t\right)
\end{gathered}
$$

where $z_{o}=a-v_{-} t$. The induced currents aren't equal. But electrons are being deposited on the plate at $a=0$ at a rate of $\rho_{o} v_{-}$per unit area so the induced current plus the charge current at the plate at $z=0$ equals the induced current at the plate at $z=a$. The current in the external circuit per unit area of the plates is

$$
J=\left(\rho_{o} v_{-} / a\right)\left(a-v_{-} t\right) \quad 0<t<a / v_{-}
$$

Fig. 4i shows the electric field within the gap as a function of $z$ at time $t=\left(a-z_{o}\right) / v_{-}$. Fig. $4 \mathrm{j}$ shows $E(a)$ and $E(0)$ as a function of $z_{o}$. Fig. $4 \mathrm{k}$ shows $E(a)$ and $E(0)$ as a function of time and Fig. 41 shows the induced current at $z=0$ and $z=a$.

The total charge (per unit cross section of collection gap) flowing through the ammeter of Fig. 3 is obtained by integrating Eq. (6.11). We get

$$
Q=\frac{a \rho_{o}}{2}
$$

Note that only half the electrons produced in the gap via ionization traverse the external circuit. For an electron created via ionization at $z$, only $z / a$ of an electron traverses the external circuit as this drifting electron moves from $z$ to 0 .

For the case where the positive ions are also mobile and, in general, $v_{+} \neq v_{-}$,

$$
J=J_{+}+J_{-}
$$




$$
\begin{aligned}
& J_{-}= \begin{cases}\left(\rho_{o} v_{-} / a\right)\left(a-v_{-} t\right) & 0<t<a / v_{-} \\
0 & \text { otherwise }\end{cases} \\
& J_{+}= \begin{cases}\left(\rho_{o} v_{+} / a\right)\left(a-v_{+} t\right) & 0<t<a / v_{+} \\
0 & \text { otherwise }\end{cases}
\end{aligned}
$$

Note that at $t=0$, the current density is independent of the gap width $a$. If $v_{+} \ll v_{-}$then the current due to the positive ions is small compared to that for electrons during the time that electrons flow. After the electrons drift out of the gap, the only current is due to positive ions. After the positive ions drift out of the gap, the total charge collected is twice that collected from electrons alone, i.e. $Q=a \rho_{o}$.

\subsection{Example 4}

We will now consider an artificial example which will be instructive later. We assume the whole gap is uniformly filled with immobile positive ions of charge density $\rho_{+}=\rho_{o}\left(\rho_{o} \ll V_{o} \epsilon / a^{2}\right)$ and mobile electrons of charge density $\rho_{-}=-\rho_{o}$ such that the net charge density within the gap is zero. This is the same as in example 3. But here we assume that the electrons to the right of $z_{r}$ (where $0<z_{r}<a$ ) drift to the left with drift velocity $v_{-}$but the electrons to the left of $z_{r}$ remain stationary. So with time, a charge sheet builds up at $z=z_{r}$. The situation at time $t=\left(a-z_{o}\right) / v_{-}$(where $\left.z_{o}>z_{r}\right)$ is shown in the first four plots of Fig. 5. The charge sheet at $z_{r}$ has charge per unit area

$$
\sigma_{r}=-\rho_{o}\left(a-z_{o}\right)
$$

The electric field in the gap is found from Gauss' Law and by requiring the potential across the gap to remain fixed at $V_{o}$. When evaluated at $z=a$ we find

$$
E(a)=\frac{V_{o}}{a}+\frac{\sigma_{r}}{\epsilon} \frac{z_{r}}{a}-\frac{\rho_{o}}{2 \epsilon a}\left(a-z_{o}\right)^{2}+\frac{\rho_{o}}{\epsilon}\left(a-z_{o}\right)
$$

The current in the external circuit per unit area of the plates is

$$
J=\left(\rho_{o} v_{-} / a\right)\left(a-z_{r}-v_{-} t\right) \quad 0<t<\left(a-z_{r}\right) / v_{-} .
$$



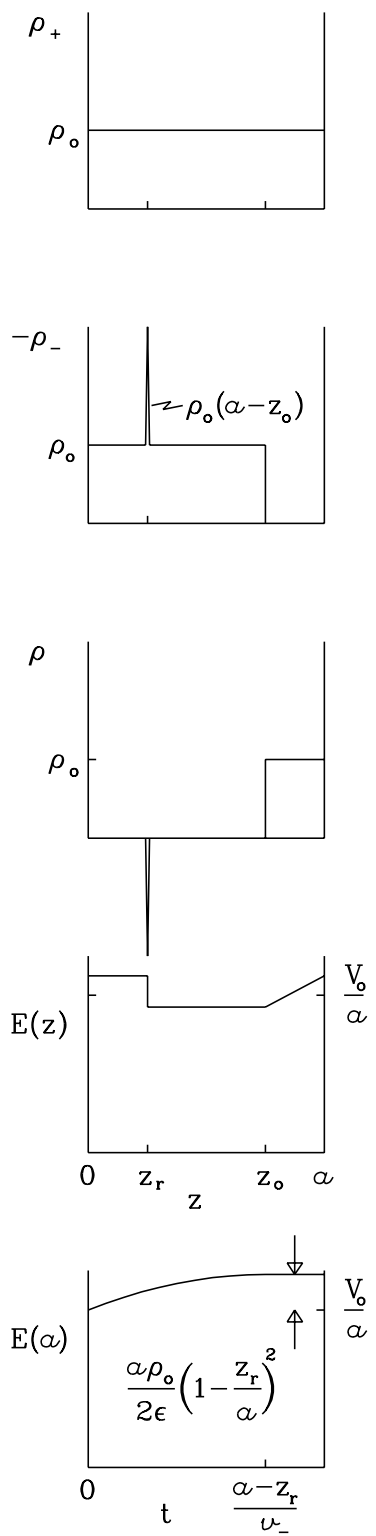

Fig. 5. Charge density and electric field for example 4 in the text.

When $t=\left(a-z_{r}\right) / v_{-}$, i.e. when $z_{o}=z_{r}$, then

$$
E(a)=\frac{V_{o}}{a}+\frac{\rho_{o}}{2 \epsilon a}\left(a-z_{r}\right)^{2}
$$

The electric field at the electrode on the right as a function of time is shown in the last plot of Fig. 5. The total charge (per unit cross section of collection 
gap) which flows through the ammeter of Fig. 3 is

$$
Q=\frac{a \rho_{o}}{2}\left(1-\frac{z_{r}}{a}\right)^{2}
$$

which is less than in example 3. (See Eq. (6.12).)

\subsection{Example 5}

One last artificial example will also be useful later. This case is the same as in the previous example but now the drifting charge does not collect at $z_{r}$. Imagine that a grid is placed at $z_{r}$ which collects all the charge as it drifts from the right and which is held at $V_{o}$ so that the electric field in the region $0<z<z_{r}$ is zero. This case is quite easy to solve and corresponds to a gap of width $a-z_{r}$ rather than the gap of width $a$ which we have considered throughout. The current in the external circuit per unit area of the plates is

$$
J=\left[\rho_{o} v_{-} /\left(a-z_{r}\right)\right]\left(a-z_{r}-v_{-} t\right) \quad 0<t<\left(a-z_{r}\right) / v_{-}
$$

The charge collected will be

$$
Q=\frac{a \rho_{o}}{2}\left(1-\frac{z_{r}}{a}\right)
$$

This is smaller than example 3, Eq. (6.12), but larger than example 4, Eq. (6.20).

\section{Charge Build-up in a Liquid Ionization Gap[21]}

For high rates of ionization the slowly drifting positive argon ions accumulate in the gap to such a degree that they significantly distort the electric field. This distortion degrades the performance of the ionization gap.

\subsection{The Scale of the Problem}

Let us consider, for the moment, a worst case condition which might actually be realized some years after turn-on at the LHC. At a luminosity of $10^{34}$ $\mathrm{cm}^{-2} \mathrm{sec}^{-1}$ there are of order 20 minimum bias interactions per $25 \mathrm{nsec}$ beam crossing. A forward calorimeter might be located $5 \mathrm{~m}$ from the interaction 
point and have fiducial coverage out to $|\eta|=4.5$. At this value of $|\eta|$ there will be 0.2 photons from $\pi^{o}$ decay per $\mathrm{cm}^{2}$ per crossing with average energy $12 \mathrm{GeV}$ incident on the surface of the calorimeter. At a depth within the calorimeter near electromagnetic shower maximum the transverse electromagnetic shower size is of order $2 \mathrm{~cm}$ in radius so that it covers an area of order $12 \mathrm{~cm}^{2}$. (We take this area to be the size of a readout tower in the EM section of the calorimeter.) So at a given point within a gap near electromagnetic shower maximum and near $|\eta|=4.5$ there will be $\sim 2$ electromagnetic showers per 25 ns beam crossing. For the typical $2 \mathrm{~mm}$ gap width of liquid argon the time to drift out the electrons is of order 400 nsec (16 beam crossings) so we can view the deposition of ionization as approximately constant in time. In section 11 we will discuss fluctuations. The positive ions drift far more slowly and can build up to appreciable levels within the gap.

The net creation rate of ion pairs per unit volume within the gap will be given by $D(z)$. We will consider only two contributions, ionization and recombination. As an approximation we assume that all charged particles traversing the gap lose only a small fraction of their energy so that the ionization is independent of $z$. We also assume the ionization is uniform in $x$ and $y$ as argued above. Also as an approximation we consider only one form of recombination, bulk recombination. As a result we take the recombination rate per unit volume to be proportional to the product of electron density and positive ion density. Thus

$$
D(z)=D_{i}-D_{r}=D_{i}-R_{r} n_{+} n_{-} .
$$

$D_{i}$ is the rate per unit volume of production of ion pairs via ionization while $D_{r}$ is the rate per unit volume of recombination of ion pairs. $R_{r}$ is the bulk recombination rate per unit volume for unit density. So we are neglecting columnar recombination as discussed in section 5.2. We also neglect for now the fact that initial (germinate) recombination is significant when the electric field is small enough. There will be instances where this neglect is not warranted and we will address this in Sections 8 and 10.

At what rate of deposition of ionization does the positive charge accumulation significantly distort the electric fields and modify the signal? To set the scale, let us start with a very qualitative argument before we begin a more precise analysis. Consider the gap in Fig. 3 with the plate at the left at electrical potential $V_{o}$ and the plate at the right at ground. Then the electric field within the gap of width $a$ is $E=V_{o} / a$ and is directed to the right. The surface charge density on each plate is $|\sigma|=\epsilon|E|=\epsilon V_{o} / a$. We will say that the electric field within the gap is significantly altered when the number of positive ions distributed across the gap equals the number of charges on the 
two plates. We will call this a critical condition. Thus

$$
\rho_{c}=e n_{c}=2|\sigma| / a=2 V_{o} \epsilon / a^{2}
$$

where $\rho_{c}$ is the critical charge density and $n_{c}$ is the corresponding critical number density where we assume that the positive ions are singly charged. If the positive ions drift at the speed $v_{+}=\mu_{+} V_{o} / a$ and, to be removed from the gap, a positive ion must drift a distance $a / 2$ on average, then the characteristic time to clear the gap is $\tau=a / 2 v_{+}=a^{2} / 2 V_{o} \mu_{+}$. We will define the critical ionization rate, $D_{c}$, as that rate of ion pair creation per unit volume equal to the rate at which the critical density of positive ions is removed from the gap, i.e.

$$
D_{c} \equiv \frac{n_{c}}{\tau}=\frac{4 V_{o}^{2} \epsilon \mu_{+}}{e a^{4}}
$$

This corresponds to a critical current density of positive ions at the plate on the right of

$$
J_{c}=e a D_{c}=\frac{4 V_{o}^{2} \epsilon \mu_{+}}{a^{3}}=\frac{2 V_{o} \mu_{+}}{a} \rho_{c}=2 v_{+} \rho_{c} .
$$

We can also define a critical recombination rate per unit volume for unit density as

$$
\left(R_{r}\right)_{c}=D_{c} / n_{c}^{2}=\frac{e}{\epsilon} \mu_{+}
$$

and a relative recombination rate per unit volume for unit density as

$$
\mathcal{R}=\frac{R_{r}}{\left(R_{r}\right)_{c}}
$$

so that

$$
D(z)=D_{i}-\mathcal{R}\left(D_{c} / n_{c}^{2}\right) n_{+} n_{-} .
$$

Finally we define the relative rate per unit volume of ionization as

$$
r \equiv \frac{D_{i}}{D_{c}}
$$

so that

$$
D(z)=D_{c}\left[r-\mathcal{R}\left(\frac{n_{+}}{n_{c}}\right)\left(\frac{n_{-}}{n_{c}}\right)\right] .
$$


In the foregoing discussion the critical values of the dynamical variables were determined in a somewhat qualitative manner but we will see in the following that these values are quantitatively just what we want.

\subsection{The Defining Equations and Constraints}

Referring again to Fig. 3 with the electric field directed to the right, the electrons drift to the left and the positive ions, much more slowly, to the right. Current conservation gives

$$
\partial_{\mu} j_{+}^{\mu}=-\partial_{\mu} j_{-}^{\mu}=e D(z)
$$

where $j_{ \pm}^{i}=\mathrm{v}_{ \pm} \rho_{ \pm}$and $j_{ \pm}^{o}=\rho_{ \pm}$. In our case $j_{ \pm}^{x}=j_{ \pm}^{y}=0$ and we use $j_{ \pm}^{z} \equiv J_{ \pm}(z)$. For $D(z)$ constant in time a steady state condition will eventually be reached so that

$$
\frac{\partial \rho_{ \pm}}{\partial t}=0 \quad \text { and } \quad \frac{\partial J_{ \pm}}{\partial z}= \pm e D(z)
$$

Since electrons do not emanate from the plate at the right (secondary emission is negligible) nor do positive ions from the plate at the left we have the boundary conditions

$$
J_{+}(0)=0 \quad \text { and } \quad J_{-}(a)=0
$$

which gives

$$
\begin{aligned}
& J_{+}(z)=e \int_{0}^{z} \mathrm{~d} z^{\prime} D\left(z^{\prime}\right)=\mathrm{v}_{+} \rho_{+}=\left(\mu_{+} E\right) \rho_{+} \\
& J_{-}(z)=e \int_{z}^{a} \mathrm{~d} z^{\prime} D\left(z^{\prime}\right)=\mathrm{v}_{-} \rho_{-}= \begin{cases}v_{-}\left|\rho_{-}\right| ; & E>E_{o} \\
\left(\mu_{-} E\right)\left|\rho_{-}\right| ; & E<E_{o}\end{cases}
\end{aligned}
$$

and

$$
J=J_{+}(z)+J_{-}(z)=e \int_{0}^{a} \mathrm{~d} z^{\prime} D\left(z^{\prime}\right)
$$

which is constant, independent of $z$, as required by charge conservation. 
Gauss' Law for our geometry gives

$$
\epsilon \frac{d E}{d z}=\rho=\rho_{+}+\rho_{-}
$$

so that

$$
E=\frac{1}{\epsilon} \int_{0}^{z} \mathrm{~d} z^{\prime} \rho\left(z^{\prime}\right)+c
$$

and the constant of integration must be adjusted to give

$$
\int_{0}^{a} \mathrm{~d} z E=V_{o}
$$

(Remember that $V_{o}$ is the potential on the electrode at $z=0$ relative to the electrode at $z=a$.) The surface charge density on the plate on the left is given by

$$
\sigma(0)=\epsilon E(0)
$$

while the surface charge density on the plate at the right is

$$
\sigma(a)=-\epsilon E(a)
$$

The electric field is zero to the left of the plane at $z=0$ and to the right of the plane at $z=a$ so the net charge contained between these two planes must be zero. This condition is already ensured by the above set of equations, i.e. it is easy to show that

$$
\sigma(0)+\sigma(a)+\int_{0}^{a} \mathrm{~d} z \rho(z)=0
$$

for all times.

\subsection{Solutions to the Steady State Equations}

In this section we will find steady state solutions which satisfy Eq. (7.7) (or Eq. (7.9)), Eq. (7.12), Eq. (7.13), Eq. (7.16), and Eq. (7.17) for various ranges 
of $r$ where $r$ is given by Eq. (7.8). These form a set of non-linear, coupled differential equations whose solutions must be found by trial and error.

When $r$ is small we expect $\rho_{+}$and $\rho_{-}$to be proportional to $r$. This suggests that $D_{r}$, i.e. the second term in Eq. (7.9), can be neglected compared to $D_{i}$. If we take $D(z)=D_{i}$, then

$$
J_{+}(z)=e z D_{i} \quad \text { and } \quad J_{-}(z)=e(a-z) D_{i}
$$

Using the definitions in Eq. (7.3), Eq. (7.4), and Eq. (7.8) we can rewrite the above as

$$
\frac{J_{+}}{J_{c}}=\left(\frac{z}{a}\right) r \quad \text { and } \quad \frac{J_{-}}{J_{c}}=\left(1-\frac{z}{a}\right) r .
$$

From Eq. (7.12) and Eq. (7.13) we see that $\left|\rho_{-}\right| \ll \rho_{+}$because $v_{-} \gg \mu_{+} E$ so we will neglect $\rho_{-}$. Eq. (7.12) can be written

$$
\frac{J_{+}}{J_{c}}=\left(\frac{z}{a}\right) r=\frac{1}{2}\left(\frac{E a}{V_{o}}\right) \frac{\rho_{+}}{\rho_{c}}
$$

and Eq. (7.15) becomes

$$
\epsilon \frac{d E}{d z}=\rho_{+}
$$

Eq. (7.23) and Eq. (7.24) yield a single differential equation

$$
4 r \frac{z}{a} \frac{d z}{a}=\frac{a E}{V_{o}} \frac{a d E}{V_{o}}
$$

which, with the constraint Eq. (7.17), can easily be solved. Note that $z / a$ is a dimensionless measure of the distance across the gap and $a E / V_{o}$ is a dimensionless measure of electric field in the gap.

The Case $r=0$

When $r=0$ there is no production of ionization in the gap, i.e. $D(z)=0$. Thus $J_{+}(z)=J_{-}(z)=0$. Also $\rho_{+}(z)=\rho_{-}(z)=0$ and $E(z)=V_{o} / a$.

The Case $0<r \ll 1$ 

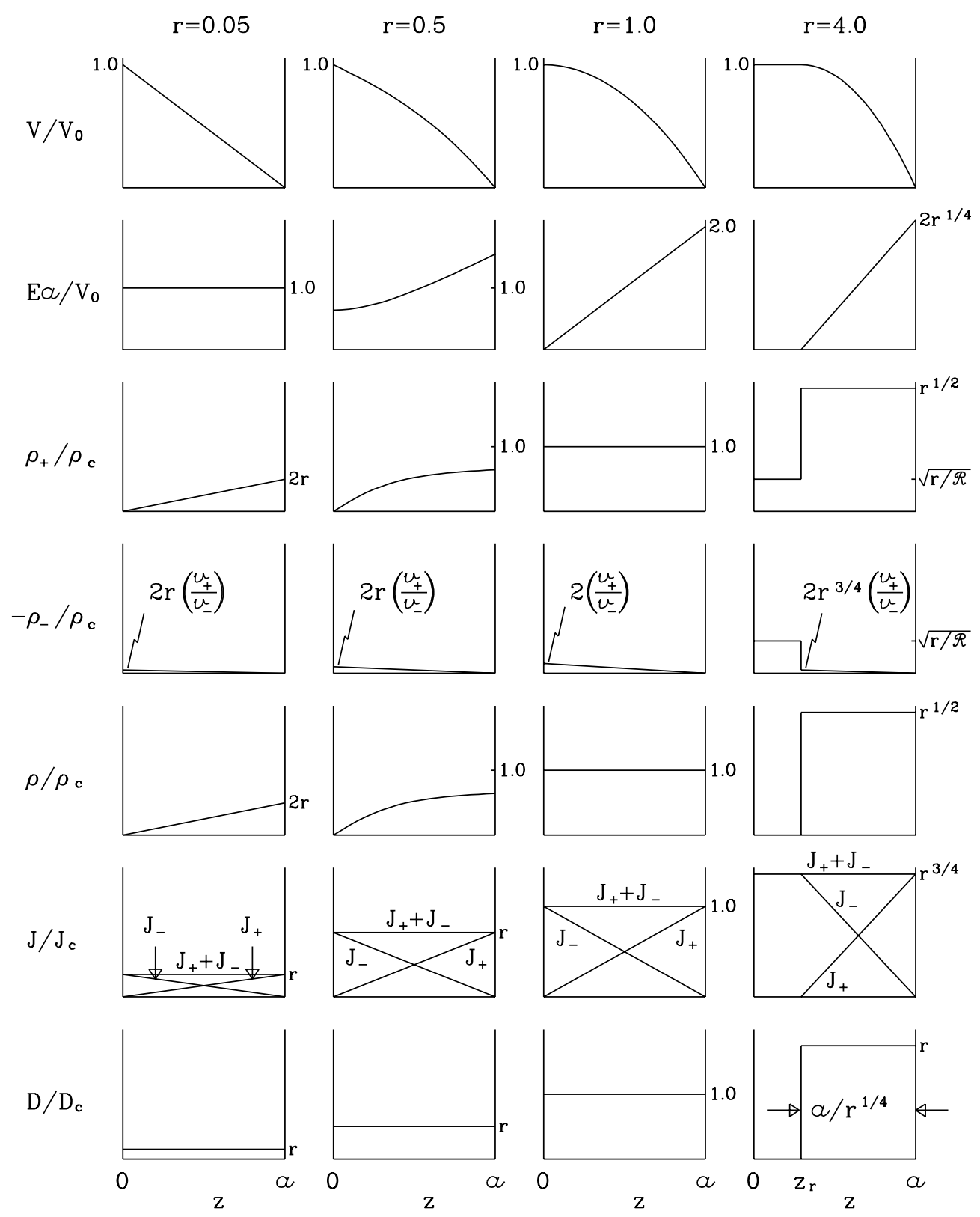

(a)

(b)

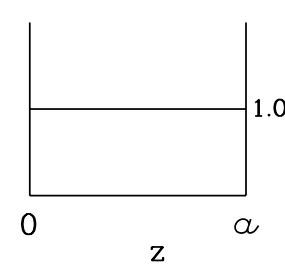

(c)

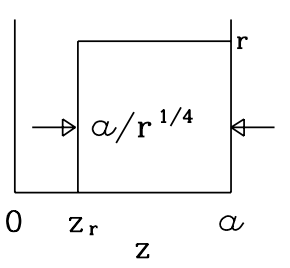

(d)

Fig. 6. Scaled potential, electric field, charge density, current density, and ionization rate in the gap for different values of the relative ionization rate $r$.

A solution of Eq. (7.25) is

$$
\left(\frac{a E}{V_{o}}\right)^{2}=k+4 r\left(\frac{z}{a}\right)^{2}
$$


For the case $r=0$ we saw that the arbitrary constant $k$ must be $k=1$. So for the case $r \ll 1$ we will try a solution $k=1+c r$ where $c$ is another arbitrary constant. Taking the square root of Eq. (7.26), expanding the right hand side to lowest order in $r$, and using the constraint Eq. (7.17) gives $k=1-4 r / 3$ and

$$
E=\frac{V_{o}}{a}\left\{1+2 r\left[(z / a)^{2}-1 / 3\right]\right\}
$$

Solutions for the other quantities of interest follow immediately. The series of plots in Fig. 6a depicts this case. Note that the approximation we made, i.e. $D_{r} \ll D_{i}$, is valid if

$$
\mathcal{R} \ll \frac{1}{4 r(z / a)(1-z / a)}\left(\frac{v_{-}}{v_{+}}\right) .
$$

The right hand side is smallest when $z=a / 2$. At this worst case value of $z$ the expression becomes

$$
\mathcal{R} \ll \frac{1}{r}\left(\frac{v_{-}}{v_{+}}\right) .
$$

\section{The Case $0<r<1$}

While the positive ions do build up to significant levels for $r$ close to one, the electrons do not. Then it is still safe to neglect the recombination rate compared to the ionization rate so we continue to assume $D(z)=D_{i}$. An exact solution of Eq. (7.25) with constraint Eq. (7.17) is

$$
\begin{aligned}
& E=\frac{V_{o}}{a} \sqrt{k+4 r(z / a)^{2}} \\
& \frac{\rho_{+}}{\rho_{c}}=\frac{2 r(z / a)}{\sqrt{k+4 r(z / a)^{2}}}
\end{aligned}
$$

where

$$
k=\frac{4}{\left[\sqrt{1+y}+\sqrt{\frac{1}{y}} \ln (\sqrt{y}+\sqrt{1+y})\right]^{2}} ; \quad y \equiv \frac{4 r}{k},
$$

a transcendental equation whose solution is plotted in Fig. 7. Note that 


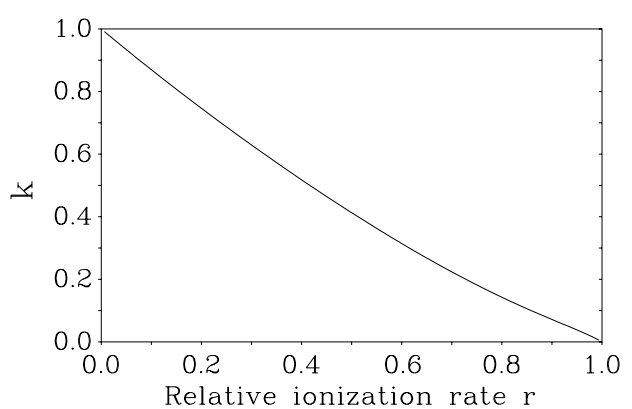

Fig. 7. The function $k(r)$ versus the relative ionization rate $r$.

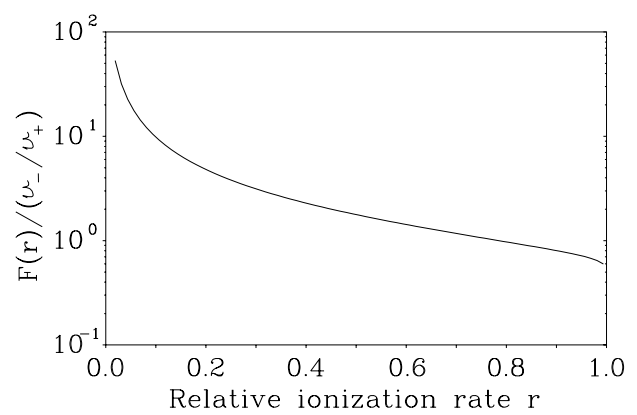

Fig. 8. The analytic expressions in the text are valid when $\mathcal{R} \ll F(r)$. This is a plot of $F(r) /\left(v_{-} / v_{+}\right)$.

$$
\begin{gathered}
\text { when } r=0 \text { then } k=1 \text { and } \\
\text { when } r=1 \text { then } k=0 \text { and } \\
\lim _{r \rightarrow 0} k(r)=1-4 r / 3
\end{gathered}
$$

An example of this case can be seen in the plots of Fig. 6b. Now we can check the validity of our approximations. Using Eq. (7.13), Eq. (7.22), and Eq. (7.4) we have

$$
\left|\rho_{-}(z)\right|=\frac{J_{-}(z)}{v_{-}}=2 \frac{v_{+}}{v_{-}}(1-z / a) r \rho_{c}
$$

if $E>E_{o}$ which is true as long as $r$ is not too close to unity. Since $v_{+} / v_{-} \ll 1$, the neglect of $\rho_{-}$relative to $\rho_{+}$is valid except for very small $z$ where $\rho_{+}$goes to zero. As $r$ approaches unity, the electric field at small $z$ falls below $E_{o}$ so that the region of small $z$, where $\rho_{-}$cannot be neglected relative to $\rho_{+}$, grows a bit.

The recombination rate is negligible if

$$
r \gg \mathcal{R}\left(\frac{\rho_{+}}{\rho_{c}}\right)\left(\frac{-\rho_{-}}{\rho_{c}}\right)=\mathcal{R} \frac{4 r^{2}(z / a)(1-z / a)}{\sqrt{k+4 r(z / a)^{2}}}\left(\frac{v_{+}}{v_{-}}\right)
$$

or

$$
\mathcal{R} \ll \frac{\sqrt{k+4 r(z / a)^{2}}}{4 r(z / a)(1-z / a)}\left(\frac{v_{-}}{v_{+}}\right) .
$$

The right hand side is the smallest when $z=z_{w}$ where

$$
z_{w}=\frac{a}{2}\left(\frac{k}{r}\right)^{\frac{1}{3}}\left[\left\{1+\sqrt{1+\frac{8}{27} \frac{k}{r}}\right\}^{\frac{1}{3}}+\left\{1-\sqrt{1+\frac{8}{27} \frac{k}{r}}\right\}^{\frac{1}{3}}\right]
$$




$$
z_{w}=\left\{\begin{array}{lll}
a / 2 & \text { when } & r \rightarrow 0 \\
0 & \text { when } & r \rightarrow 1
\end{array}\right.
$$

At this worst case value of $z$, the expression becomes

$$
\mathcal{R} \ll F(r)
$$

where $F(r) /\left(v_{-} / v_{+}\right)$is plotted in Fig. 8 .

\section{The Case $r=1$}

Continuing the solutions of the previous subsection, Eq. (7.30) and Eq. (7.31), to $r=1$ gives

$$
\begin{aligned}
& E=\frac{V_{o}}{a} \frac{2 z}{a} \\
& \frac{\rho_{+}}{\rho_{c}}=1 \\
& \frac{\rho_{-}}{\rho_{c}}=2 \frac{v_{+}}{v_{-}}(1-z / a)
\end{aligned}
$$

Again this is the approximate solution, shown in Fig. 6c, which is valid for $\left|\rho_{-}\right| \ll \rho_{+}$. Further we must assume

$$
\mathcal{R} \ll \frac{1}{2(1-z / a)}\left(\frac{v_{-}}{v_{+}}\right)
$$

This solution is clearly not correct very near $z=0$ where the electric field falls below $E_{o}$.

\section{The Case $r>1$}

Here the approximations leading to Eq. (7.25) are no longer valid and we must use the full set of non-linear, coupled differential equations with constraints. The first order solution for this case is shown in Fig. 6d where

$$
z_{r}=a\left(1-\frac{1}{r^{\frac{1}{4}}}\right)
$$

The most striking feature is that for $z<z_{r}$ the electric field in the gap is (nearly) zero. We will say that this part of the gap has "closed down". In this 
region the positive and negative number densities are equal, i.e. $n_{+}=n_{-}$, so the net charge density, $\rho$, is zero. Rather than drifting out of the gap, the ions in this region are recombining at the same rate they are produced. The net ion creation rate, $D(z)$, is zero in this region. Nevertheless there are free ions in this region. In fact there is a large reservoir of highly mobile electrons.

The rest of the gap behaves very much like the case $r=1$ but scaled by the smaller effective gap size. Note also that the maximum value of the electric field occurs at $z=a$ and has a value

$$
E(a)=2 r^{\frac{1}{4}} \frac{V_{o}}{a}
$$

As a practical matter it is important to note that for $r>1$, the electric field at $z=a$ is at least twice the nominal field of $V_{o} / a$. For many electrode structures it is not possible to raise the electric field to such high values without inducing breakdown. Lowering $V_{o}$ would increase $r$, exacerbating the signal degradation problem to be discussed shortly but at least lowering the maximum electric field as $\sqrt{V_{o}}$.

\section{Higher Order Corrections}

The solutions obtained so far are approximate. We explained in section 4 how we would treat the drift velocities in order to obtain analytic solutions. For the cases $r<1$ and $r=1$ we have, in addition, assumed that we could neglect the charge density due to electrons, $\rho_{-}$, in comparison to $\rho_{+}$, i.e. we assumed $\rho \simeq \rho_{+}$. Further for the case $r>1$ we have neglected $\rho_{-}$relative to $\rho_{+}$for $z>z_{r}$. As a next order approximation we have estimated the effect of the small value of $\left|\rho_{-}\right|$on the solutions for the case $r>1$. This is indicated in Fig. 9. The quantitative values of these higher order corrections are needed for the calculations of Section 9 .

As can be seen from Fig. 9 the electric field in the region $z<z_{r}$ is quite small. We expect initial recombination to be significant here and it modifies some of the values in Fig. 9. Using the parameterization for initial recombination from Section 5.3 for the region $z<z_{r}$ we find

$$
\frac{\rho_{+}}{\rho_{c}}=-\frac{\rho_{-}}{\rho_{c}}=\sqrt{\frac{r P(E)}{\mathcal{R}}}=\sqrt{\frac{r}{\mathcal{R}} \frac{E}{E_{2}}} \sqrt{\ln \left(1+E_{2} / E\right)}
$$



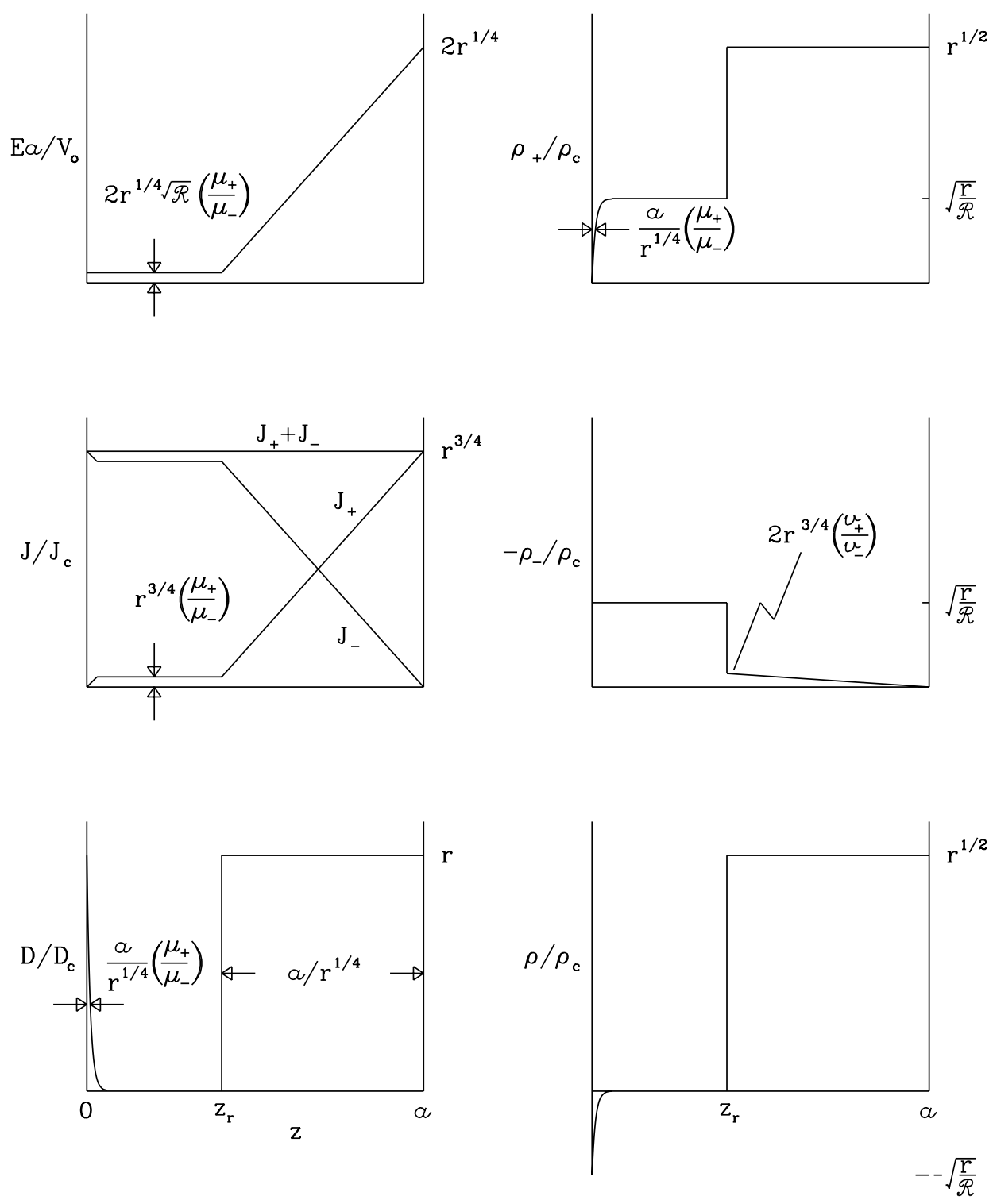

Fig. 9. Higher order corrections to the scaled electric field, current density, ionization density, and charge densities.

and using $J_{-} / J_{c}=r^{3 / 4}$ we derive the transcendental equation

$$
\frac{E a}{V_{o}}=\frac{1}{\ln ^{1 / 3}\left(1+E_{2} / E\right)}\left(2 \frac{\mu_{+}}{\mu_{-}}\right)^{\frac{2}{3}}\left(\mathcal{R} \sqrt{r} \frac{E_{2} a}{V_{o}}\right)^{\frac{1}{3}}
$$

An iterative solution converges quickly because for $E \ll E_{2}$ the logarithm to 
a small power varies slowly with $E$. We then can show that

$$
-\frac{\rho_{-}}{\rho_{c}}=\frac{\rho_{+}}{\rho_{c}}=\ln ^{1 / 3}\left(1+E_{2} / E\right)\left(\frac{2}{\mathcal{R}} \frac{\mu_{+}}{\mu_{-}} / \frac{E_{2} a}{V_{o}}\right)^{\frac{1}{3}} r^{\frac{7}{12}}
$$

Note that the product of $E a / V_{o}$ and $\rho_{-} / \rho_{c}$ is independent of $\mathcal{R}$ and $E_{2}$ and is the same as for the case with no initial recombination in Fig. 9. For $r$ near unity

and using parameter values to be discussed later we find $\ln ^{1 / 3}\left(1+E_{2} / E\right) \sim$ 1.7. So with initial recombination and $r$ near unity, $E a / V_{o}$ is larger (and $\rho_{-} / \rho_{c}$ is smaller) than the case with no initial recombination by a factor between approximately 3 and 10 .

\section{Degradation of the Signal in the Charge Build-up Environment}

The source of ionization in sections 7 and 8 is from the approximately steady showering of $\pi^{o}$ decay photons from minimum bias events. These photons have $\left\langle p_{T}\right\rangle \sim 0.3 \mathrm{GeV} / \mathrm{c}$ which is very small on the scale of the high $p_{T}$ physics goals of the high luminosity hadron colliders. We now want to investigate what happens when a high energy event comes in on top of this steady state background. Imagine, for instance, that a jet of $p_{T}=100 \mathrm{GeV}$ strikes the calorimeter. Ionization in excess of the steady state value will be produced in the gap. The electric field will cause this excess charge to drift out of the gap. So later the ionization will return to the steady state value. The current through the external circuit will increase above its steady state value while the excess ionization drifts out of the gap. It is this current in excess of the steady state value which constitutes the signal we wish to measure.

It is useful to get a feeling for the magnitudes of the quantities we are dealing with. For a conventional EM calorimeter with $2 \mathrm{~mm}$ lead absorber plates, $2 \mathrm{~mm}$ liquid argon gaps, and $2 \mathrm{kV}$ across the gap, (we arbitrarily assume $\mu_{+}=0.06$ $\left.\mathrm{mm}^{2} / \mathrm{Vs}\right)$ we calculate $\rho_{c}=13.5 \mathrm{pC} / \mathrm{mm}^{3}, n_{c}=8.5 \times 10^{7}$ ions $/ \mathrm{mm}^{3}$, and $J_{c}=1.62 \mathrm{nA} / \mathrm{mm}^{2}$.

The roughly constant background current in a $2 \mathrm{~mm}$ gap at EM shower maximum due to minimum bias events at $|\eta|=4.5$ and luminosity of $10^{34} \mathrm{~cm}^{-2}$ $\sec ^{-1}$ is about $J=27 \mathrm{nA} / \mathrm{mm}^{2}$ or $37 \mu \mathrm{A}$ in the area of $1 R_{M}$. (The rms fluctuation in the background current in the same area in the gap at EM shower maximum is about $5 \mu \mathrm{A}$. See Section 11.)

For a photon of energy $100 \mathrm{GeV}$ there are about $20 \times 10^{6}$ ions produced in the gap at shower maximum. These ions produce an initial current of about 8 $\mu \mathrm{A}$. The area covered by this EM shower is contained within a radius of order 
the Moliere radius which is $R_{M}=21 \mathrm{~mm}$.

In section 6 we discussed several examples where different patterns of ionization produced current waveforms in an external circuit when the ions drifted in the gap. Only example 3 was apparently relevant. The others were for pedagogic purposes. The last two examples will be seen to be of interest to us now. In the examples of section 6 there were no charges in the gap before the event that caused the ionization. We now wish to consider a small transient signal on top of a large steady-state current.

We assume that the electron drift velocity is independent of electric field for all but the smallest values of electric field. That is, referring to Fig. 2, we assume that $E_{o} \ll V_{o} / a$. Now consider the cases $0<r<1$ of section 7.3. The electrons from the instantaneous ionization deposited by a high energy shower on top of the steady state ionization from minimum bias events will drift out of the gap in exactly the same way as in example 3 of section 6.3 . While the electric field in the gap is quite different from the case considered in section 6.3 (and displayed in Fig. 4i) the change in the electric field (from that shown in Fig. 6 a or $6 \mathrm{~b}$ ) due to the instantaneous deposition of ionization is the same as the change in Fig. 4i from $V_{o} / a$. So the time-varying current density on top of the steady-state background is the same as in section 6.3, i.e.

$$
J(t)=\left(\rho_{o} v_{-} / a\right)\left(a-v_{-} t\right) \quad 0<t<a / v_{-}
$$

At time $t=0$ this current density is $J(0)=\rho_{o} v_{-}$. We will now define the signal $S(t)$ for all values of $r$ as

$$
S(t) \equiv J(t) / \rho_{o} v_{-}
$$

Then the signal for $r<1$ is

$$
S(t)= \begin{cases}1-t / t_{d} & 0<t<t_{d} \\ 0 & \text { otherwise }\end{cases}
$$

where

$$
t_{d} \equiv a / v_{-}
$$

is the drift time for electrons across the gap. This will be the standard signal to which we will refer all others. For $r>1$ the signal is less than this standard signal. We now develop an approximate expression for the signal when $r>1$.

As outlined above, we assume that at $t=0$ there is some instantaneous ionization deposited on top of the steady-state charge density $\rho$ (see Fig. 6d) 
leaving excess charge density $\delta \rho_{+}=-\delta \rho_{-}=\rho_{o}$ such that $\delta \rho\left(=\delta \rho_{+}+\delta \rho_{-}\right)=0$ at time $t=0$. We will assume that the positive ions remain fixed on the time scales of interest to us here. In the region $z>z_{r}$, the electrons drift to the left at velocity $v_{-}$independent of any changes in the electric field because $E(z)>E_{o}$. In the region $z<z_{r}$ the electrons drift so slowly that, on the time scale of interest, no charges drift a significant distance from $z=z_{r}$. So a charge sheet will build up at $z=z_{r}$ of magnitude $\sigma_{r}$. At some time $t$ after the deposition of the instantaneous ionization, the charge density in the gap, in excess of the steady-state values, is as shown in the third plot of Fig. 5 except that the size of the delta function charge build-up at $z=z_{r}$ will not, in general, be the same. As in section 6 , we have $z_{o}=a-v_{-} t$. Due to this excess charge density the electric fields in the gap are modified as follows:

$$
\delta E= \begin{cases}E_{g} & 0<z<z_{r} \\ E_{g}+\sigma_{r} / \epsilon & z_{r}<z<z_{o} \\ E_{g}+\sigma_{r} / \epsilon+\left(z-z_{o}\right) \rho_{o} / \epsilon & z_{o}<z<a\end{cases}
$$

where $E_{g}$ and $\sigma_{r}$ are to be determined. We require, as always, that the potential across the gap be $V_{o}$. In this case where the steady-state charge distribution already establishes the electrical potential we have $\int_{0}^{a} \delta E \mathrm{~d} z=0$. Solving for $E_{g}$ we get

$$
E_{g}=-\frac{\sigma_{r}}{\epsilon a}\left(a-z_{r}\right)-\frac{\rho_{o}}{2 \epsilon a}\left(a-z_{o}\right)^{2}
$$

where, from the first line of Eq. (9.5), we have

$$
\delta E\left(z<z_{r}\right)=E_{g}
$$

The charge build-up $\sigma_{r}$ at $z=z_{r}$ comes from two contributions, 1) the electrons in the region $z<z_{r}$ drifting slowly to the left at velocity $\mu_{-} E$ leaving behind positive charge at $z_{r}$ and 2) electrons drifting from the region $z>z_{r}$ at velocity $v_{-}$piling up at $z_{r}$. We now solve for this charge build-up $\sigma_{r}(t)$.

$$
\begin{aligned}
d \sigma_{r} & =\left[\mu_{-}\left(E\left(z_{r}\right)+\delta E\left(z_{r}^{-}\right)\right)\left|\rho_{-}\left(z_{r}^{-}\right)\right|-v_{-}\left|\rho_{-}\left(z_{r}^{+}\right)\right|\right] d t \\
& =\left[\mu_{-} \sqrt{\frac{r}{\mathcal{R}}} \rho_{c}\left(E\left(z_{r}\right)+\delta E\left(z_{r}^{-}\right)\right)-v_{-}\left(2 r^{3 / 4}\left(\frac{v_{+}}{v_{-}}\right) \rho_{c}+\rho_{o}\right)\right] d t \\
& =\left[\mu_{-} \sqrt{\frac{r}{\mathcal{R}}} \rho_{c} E_{g}-v_{-} \rho_{o}\right] d t
\end{aligned}
$$

In the second step above we assumed that $\rho_{o} \ll \rho_{c}$ and in the last step the steady state parts have cancelled as they must when $\rho_{o}=0$. We must solve 
the differential equation

$$
\frac{d \sigma_{r}}{d t}=-\left[\mu_{-} \sqrt{\frac{r}{\mathcal{R}}} \rho_{c} \frac{a-z_{r}}{\epsilon a}\right] \sigma_{r}-\left[\mu_{-} \sqrt{\frac{r}{\mathcal{R}}} \rho_{c} \rho_{o} \frac{v_{-}^{2}}{2 \epsilon a}\right] t^{2}-v_{-} \rho_{o}
$$

with boundary condition $\sigma_{r}(0)=0$. It is easy to show that the signal, subtracting off the steady-state part, is

$$
\begin{aligned}
& S(t)=1-r^{\frac{1}{4}} t / t_{d}-\frac{z_{r}}{a} e^{-\left(r^{\frac{1}{4}} / w\right)\left(t / t_{d}\right)}+w \frac{z_{r}}{a}\left(1-e^{-\left(r^{\frac{1}{4}} / w\right)\left(t / t_{d}\right)}\right) \\
& \text { when } 0 \leq \frac{t}{t_{d}} \leq \frac{1}{r^{\frac{1}{4}}}
\end{aligned}
$$

and

$$
S(t)=\frac{z_{r}}{a}\left(w e^{\frac{1}{w}}-(w+1)\right) e^{-\left(r^{\frac{1}{4}} / w\right)\left(t / t_{d}\right)} \quad \text { when } \quad \frac{t}{t_{d}}>\frac{1}{r^{\frac{1}{4}}}
$$

where

$$
\begin{aligned}
& w \equiv \frac{\sqrt{\mathcal{R}}}{2 R_{-}} \\
& R_{-} \equiv \frac{\mu_{-}\left(V_{o} / a\right)}{v_{-}} \equiv \frac{u_{-}}{v_{-}} .
\end{aligned}
$$

(See Fig. 2 for an interpretation of $u_{-}$.) $S(t)$ is plotted in Fig. 10 for the case $r=16$ and for several values of $w$. The standard signal for $r \ll 1$ is shown for reference.

We now want to be more specific about the measured signal. Two extremes should serve. The first extreme is close to a situation which could arise at a high luminosity hadron collider. One might trigger on an event and gate or shape the calorimeter signal just at the beginning of the signal charge collection time. Then the signal would be proportional to the current through the ammeter of Fig. 3 at time $t=0$. We call this the "initial current signal" $S_{i}$. The second extreme is one we're used to at lower luminosities. It's the case where the charge collection time is long enough to collect all of the induced charge due to electrons. We will completely ignore the small contribution from the much slower-moving positive ions. That is, we will accumulate charge for the time that it takes the signal electrons to drift out of the active part of the gap $\left(z_{r}<z<a\right)$, i.e. we will evaluate the integrated charge at time

$$
t_{o}=\frac{a-z_{r}}{v_{-}}=\frac{a}{r^{1 / 4} v_{-}}
$$




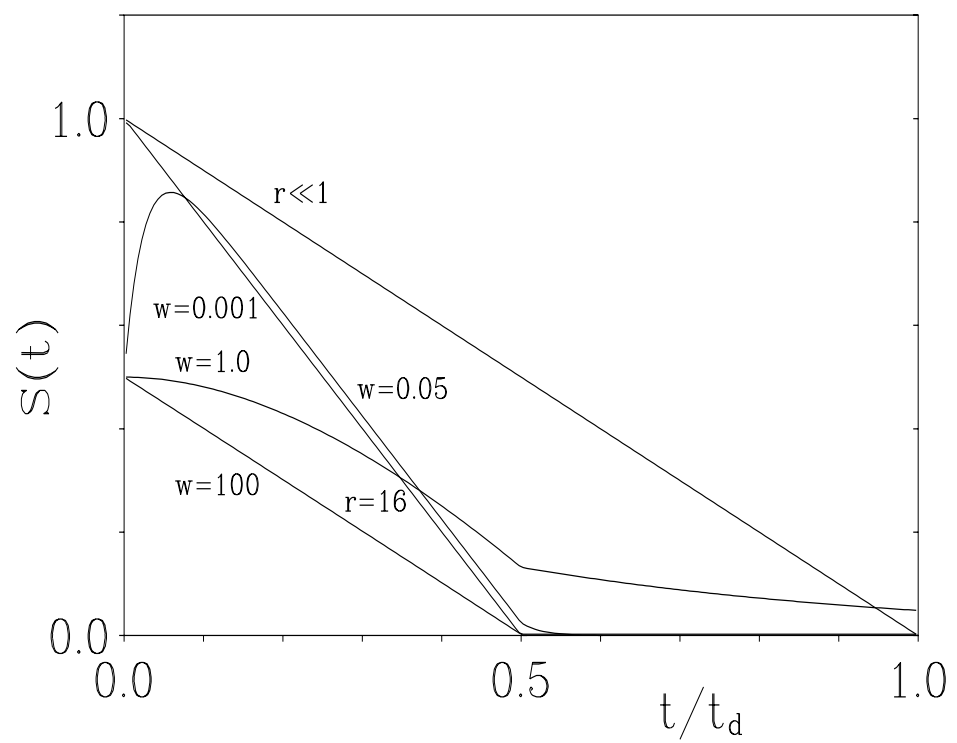

Fig. 10. Signal $\mathrm{S}(\mathrm{t})$ for $r=16$ and several values of $w$. The usual case of $r \ll 1$ is shown for comparison.

i.e. when $z_{o}=z_{r}$. We call this the "integrated charge signal" $S_{q}$.

We see that the initial current signal, using Eq. (9.10) for $r>1$, is

$$
S_{i}= \begin{cases}1 & \text { for } r \leq 1 \\ \frac{1}{r^{1 / 4}} & \text { for } r>1\end{cases}
$$

By integrating Eq. (9.10) we find

$$
S_{q}=\frac{1}{r^{1 / 4}}\left[1-\frac{z_{r}}{a} f(w)\right]
$$

where

$$
f(w)=2 w\left\{w-(w+1) e^{-1 / w}\right\}
$$

The limiting values of $f(w)$ are

$$
\lim _{w \rightarrow 0} f(w)=2 w^{2}
$$

and

$$
\lim _{w \rightarrow \infty} f(w)=1
$$


which gives

$$
\lim _{w \rightarrow 0} S_{q}=\frac{1}{r^{1 / 4}}
$$

and

$$
\lim _{w \rightarrow \infty} S_{q}=\frac{1}{r^{1 / 2}}
$$

The limit $w \rightarrow 0$ occurs when the relative recombination rate $\mathcal{R}$ is small so the reservoir of ions and electrons in the region $0<z<z_{r}$ is large. In this case the charge sheet, $\sigma_{r}$, accumulated at $z_{r}$ is just the right amount to keep $E_{g}$, the incremental field in the region $0<z<z_{r}$ near zero. Nevertheless it is large enough to speed up the reservoir of electrons a small amount leaving behind at $z_{r}$ a small positive charge. This small positive charge at $z_{r}$ is more than cancelled by the electrons that drift from the region $z>z_{r}$ and collect there. The net charge $\sigma_{r}\left(t_{o}\right)$ is half the electron charge originally deposited by the instantaneous ionization of density $-\rho_{o}$ in the region $z_{r}<z<a$. This limit is similar to the artificial example in section 6.5.

The limit $w \rightarrow \infty$ occurs when the relative recombination rate $\mathcal{R}$ is large so the reservoir of ions and electrons in the region $0<z<z_{r}$ is small. While $E_{g}$ is now positive there are an insignificant number of electrons in this region to move away and expose the remaining positive ions. So all the electrons drifting from the region $z_{r}<z<a$ build up at $z_{r}$. This limit is similar to the artificial example in section 6.4 .

Fig. 11 shows the signals, $S_{i}$ and $S_{q}$, as a function of $r$. In the simple approximation we have made, the signals are constant for $r<1$. But for $r>1$ the signals drop off steeply. For the initial current signal $S_{i}$ in the upper plot, the highest curve at $r$ near 1 applies, independent of $\mathcal{R}$. The degree to which the integrated charge signal $S_{q}$ drops off depends on the value of $\mathcal{R}$ as can be seen in the lower figure. The full range of possibilities falls within the range of the curves shown.

Although the initial current signal $S_{i}$ falls off steeply for $r>1$ (independent of $w)$, this is an idealization for values of $w \ll 1$. Note in Fig. 10 that $S(t)$ rises steeply right after $t=0$ from its initial value $S_{i}$ for $w=0.05$. For $w=0.001$ the initial rise is so steep that it cannot be seen on the plot. For very small values of $w$ a practical measurement device would record a value of $S_{i}$ closer to 1.0 , even for the exceptionally large value $r=16$ shown in the plot. So no steep fall in $S_{i}$ above $r=1$ would be measured for small $w$. However the likely value of $w$ is large enough that there is no rise in $S(t)$ near $t=0$. Thus mismeasurement is not an issue. 

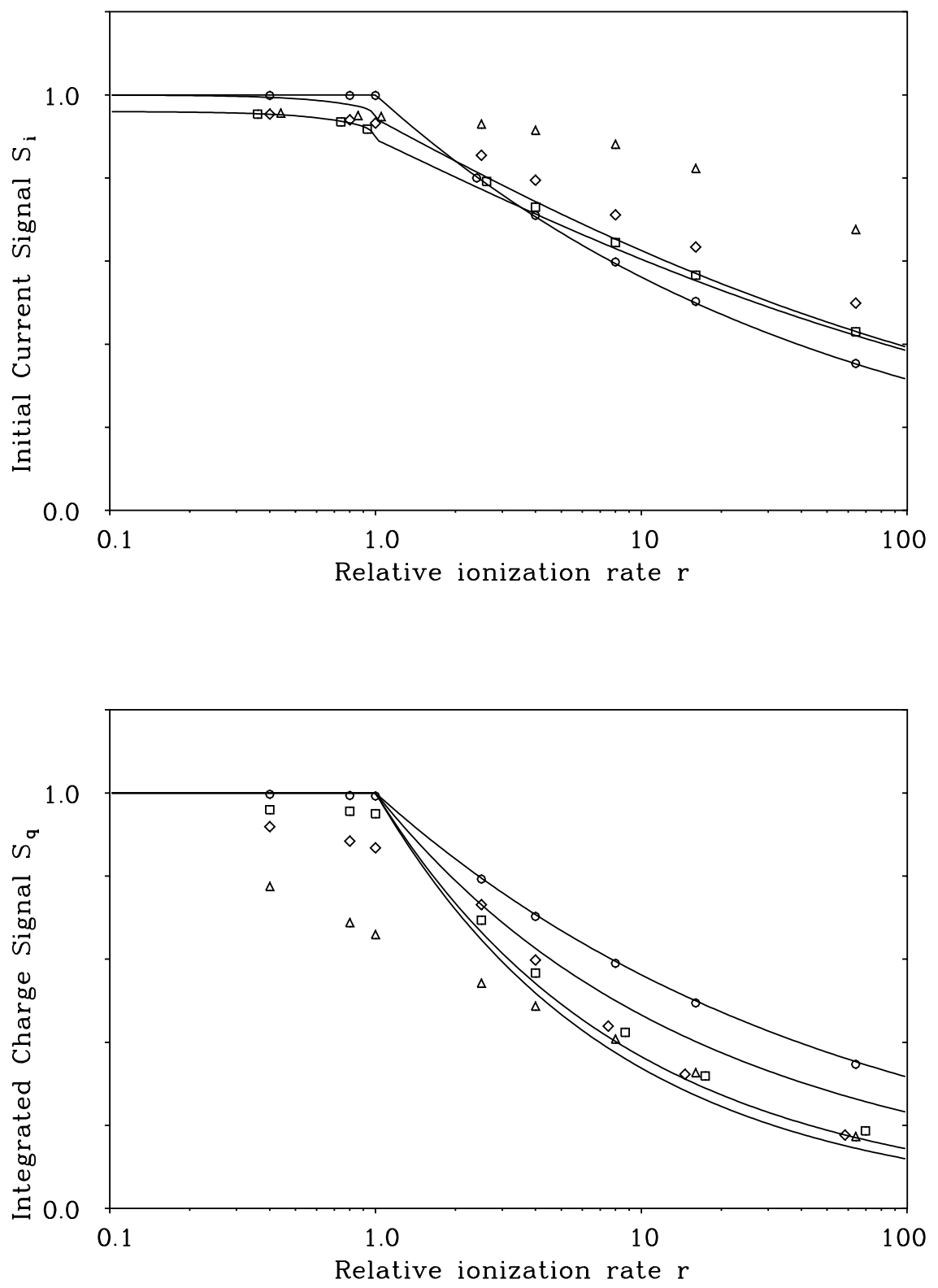

Fig. 11. Signals $S_{i}$ and $S_{q}$ as a function of the relative ionization rate $r$ from the analytic calculations (lines) and from simulations (data points). A few data points are displaced horizontally by a small amount so that they do not overlap. Four simulation cases are shown. 1) The ideal conditions closely corresponding to the analytic calculations (circles). 2) Realistic electron velocity and initial recombination vs. electric field (squares). 3) Same as case 2 but the relative recombination rate $\mathcal{R}$ is increased to the modestly high value of 5,000 (diamonds). 4) Recent measured value of relative recombination rate $\mathcal{R}$ (triangles). In the upper plot the three curves are Eq. (9.15), Eq. (9.22), and Eq. (9.23) (from highest to lowest near $r=1$ ) while in the lower plot the four curves are Eq. (9.16) for values of $w=0.08,0.67,2.95$, and 7.21 from highest to lowest in the region $r>1$. 
Had we been confident that the steady state charge densities would not affect the initial current signal $S_{i}$ then we could have predicted Eq. (9.15) without solving the full problem. But having done so, we now have confidence that the large reservoir of highly mobile electrons at $0<z<z_{r}$ does not affect $S_{i}$ except when $w$ approaches zero. Armed with this observation it is straightforward to improve the estimate of the initial current signal $S_{i}$ to account for a more realistic dependence of the electron velocity on electric field. Assuming we know $\mathrm{v}_{-}(E)$, adopting our zeroth order assumption that the excess charge density $\rho_{o}$ associated with the signal is uniform across the gap, ignoring the steady state background, generalizing Eq. (6.5), and using the definition in Eq. (9.2) we find

$$
S_{i}=\frac{<\mathrm{v}_{-}>}{v_{-}}=\frac{1}{a v_{-}} \int_{0}^{a} \mathrm{~d} z \mathrm{v}_{-}(E)=\frac{1}{a v_{-}} \int_{0}^{a} \mathrm{~d} E \frac{\mathrm{d} z}{\mathrm{~d} E} \mathrm{v}_{-}(E)
$$

For $r>1$ there are regions of the gap where $\mathrm{v}_{-}(E)$ is zero (since $E=0$ ) so that part of the gap does not contribute to the integral. For the parts of the gap that do contribute, the weighting is now improved.

A further improvement is to include initial recombination, say as given by Eq. (5.2). Now the initial current signal is approximated by

$$
S_{i}=\frac{1}{a v_{-}} \int_{0}^{a} \mathrm{~d} z \mathrm{v}_{-}(E) P(E)
$$

\section{Simulations}

The analytic calculations presented so far require approximations. We have performed extensive computer simulations of the charge build-up in order to check the calculations, to estimate the effect of some of the approximations, and to extend the results beyond the range of validity of the analytic calculations. For these simulations, the nature of the approximations is different from the analytic case, giving a feeling for the accuracy of the results.

The computer model assumes the same linear geometry, i.e. that the only spatial variable is z. (See the beginning of Section 6.) The liquid argon gap was divided into $n$ equal segments each of width $a / n$ where $a=2 \mathrm{~mm}$. The positive and negative charges in each segment changed in each time step due to 1) ionization, 2) recombination, 3) charges drifting in from an adjacent segment on one side, and 4) charges drifting out to an adjacent segment on the other side. The time step was chosen small enough that the faster negative 
charges, the electrons, would drift a quarter of one segment in one time step at the nominal velocity $v_{-}$. Diffusion was neglected as before.

Convergence was monitored by comparing the net ionization rate (including recombination) with the current density at $z=0$ and at $z=a$, i.e. $J_{-}(0)$ and $J_{+}(a)$. These typically agreed to at least three significant digits and sometimes far better. In addition the distributions $\rho_{+}(z), \rho_{-}(z), J_{+}(z), J_{-}(z)$, and $E(z)$ were monitored periodically

Several methods to speed convergence of the simulations were tried. The first was to start the simulations with the analytic results. The second relied on yet another scaling relation. In the region $z>z_{r}$ where $\rho_{+} \rho_{-}$is small enough that the bulk recombination rate is negligible, we have $\rho_{-} \sim 1 / \mathrm{v}_{-}$so $J_{-}=\rho_{-} \mathrm{v}_{-}$is invariant to changes in $\mathrm{v}_{-}$. In the region $z<z_{r}$ where the bulk recombination rate is large, $n_{+} \sim 1 / \sqrt{\mathcal{R}}$ and $n_{-} \sim 1 / \sqrt{\mathcal{R}}$ so $D_{r}=\mathcal{R}\left(n_{+} / n_{c}\right)\left(n_{-} / n_{c}\right)$ is invariant to changes in $\mathcal{R}$. Also $E\left(z<z_{r}\right) \sim \sqrt{\mathcal{R}} / \mu_{-}$so $\mathrm{v}_{-}=\mu_{-} E \sim \sqrt{\mathcal{R}}$ and $J_{-}=\rho_{-} \mathrm{v}_{-}$is invariant to simultaneous changes in $\mathrm{v}_{-}$and $\mathcal{R}$. $J_{+}$is almost zero anyway. So scaling $\mathrm{v}_{-}$, i.e $v_{-}$and $\mu_{-}$, and $\mathcal{R}$ by the same factor leaves the currents $J_{+}$and $J_{-}$unchanged. If $\mathcal{R}$ and $\mathrm{v}_{-}$are scaled down by some large factor, such as 1000, then the iterations converge 1000 times faster. Neither of these techniques was used in the simulations described here.

The third technique, which we did use, was to divide the gap into $n=64$ segments. The starting conditions were as though the ionization source was turned on abruptly (i.e. no free charges initially) and the iterations proceeded as in a real experiment. After convergence the gap was then divided into $n=128$ segments and iterations continued with starting conditions from the converged results at $n=64$. The number of segments was increased by a factor of 2 several more times until $n=1024$ converged.

After the steady state condition converged a small excess charge was introduced uniformly across the gap to simulate a signal and further iterations measured the induced current due to this signal on top of the constant background current. The resultant induced current was measured by recording the electric field at the electrode at $z=a$ at each iteration after the signal. A pedestal value was subtracted from these recorded electric field values. The pedestal was determined from an average over the last 2000 iterations before the signal of the electric field at the electrode at $z=a$. Because convergence was reached before the signal was deposited, this pedestal electric field did not change with each iteration. Nevertheless any small, linear change in the pedestal was also determined and extrapolated to the iterations following the signal deposition. This would make a small correction to the pedestal value and it was this corrected pedestal which was subtracted, iteration by iteration.

The first simulations of this sort mimicked the analytic calculations as closely 

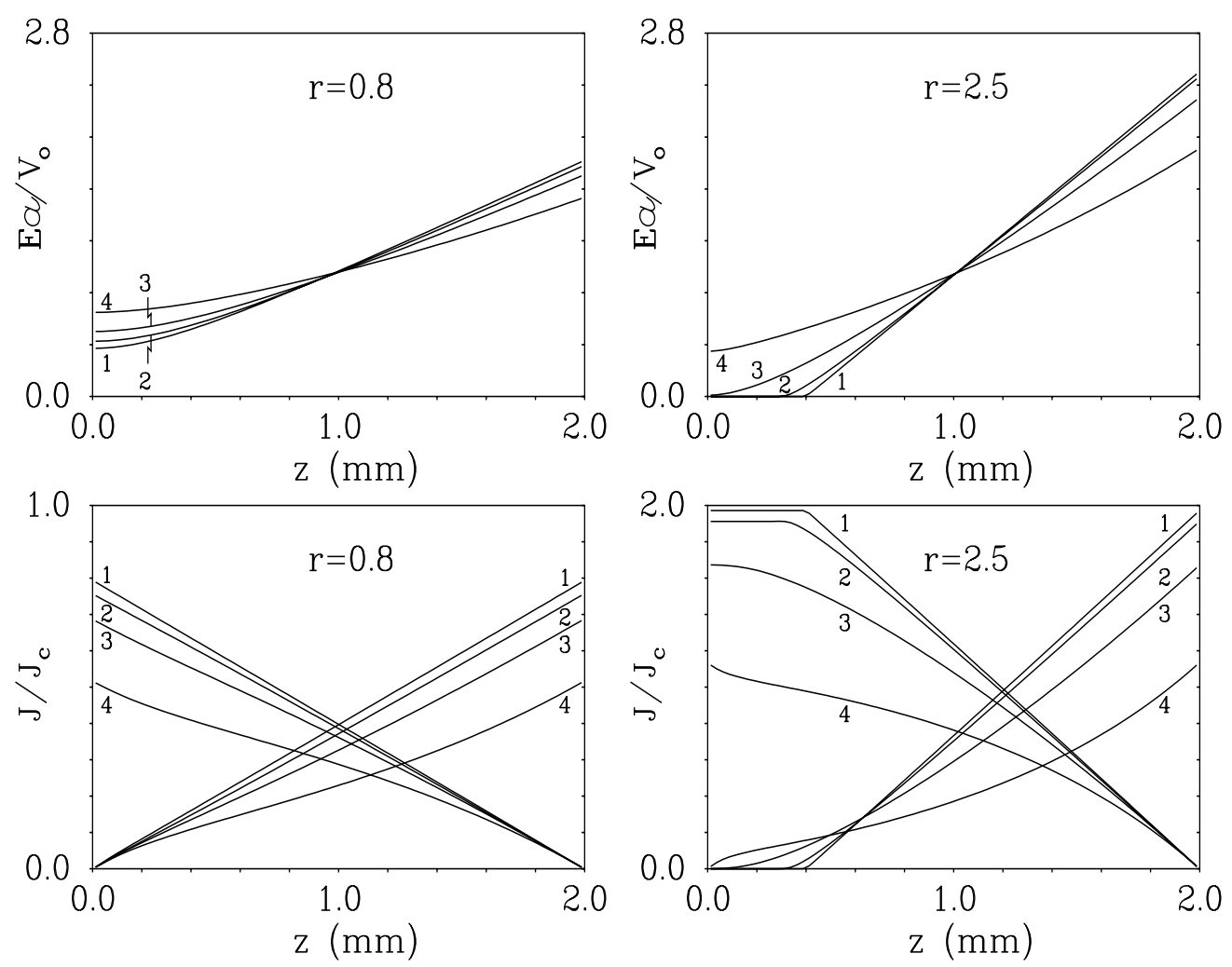

Fig. 12. Scaled electric field and current density for $r=0.8$ on the left and $r=2.5$ on the right. Simulation cases 1, 2, 3, and 4 are the same as in the previous figure caption.

as possible as a check of the calculations. Agreement was excellent. Fig. 12 shows the resultant steady state current density and electric field scaled as in previous figures. This is called case 1 in the figure. The simulation converged slowly for $r=2.5$ in the region of very small electric field as we might expect.

But in the computer simulation the electric field dependence of the drift velocity for electrons can be more accurately modelled to match the data. We employed a power law parameterization inspired by Ref. [6] as follows:

$$
\mathrm{v}_{-}= \begin{cases}A\left(E / E_{0}\right) & 0<E<E_{0} \\ B\left(E / E_{1}\right)^{a} & E_{0}<E<E_{1} \\ B\left(E / E_{1}\right)^{b} & E_{1}<E\end{cases}
$$

The parameter values are given in Table III. Initial recombination was modelled via Eq. (5.2) with the only parameter $E_{2}$ also given in Table III.

The more realistic electron velocity and initial recombination is case 2 in Fig. 12. Here convergence in the region of low electric field is much better. 
Table III

Simulation Parameters

\begin{tabular}{ccccccccccc} 
Case & $\mathcal{R}$ & $a$ & $b$ & $A$ & $B$ & $E_{0}$ & $E_{1}$ & $E_{2}$ & $w$ \\
& & & \multicolumn{5}{c}{$(\mathrm{mm} / \mu \mathrm{s})$} & \multicolumn{3}{c}{$($ Volts $/ \mathrm{mm})$} \\
1 & 256 & 0.0 & 0.0 & 5.0 & 5.0 & 10 & 10,000 & 0.001 & 0.08 \\
2 & 256 & 0.365 & 0.1 & 1.2 & 6.4 & 20 & 2,000 & 84 & 0.67 \\
3 & 5,000 & 0.365 & 0.1 & 1.2 & 6.4 & 20 & 2,000 & 84 & 2.95 \\
4 & 30,000 & 0.365 & 0.1 & 1.2 & 6.4 & 20 & 2,000 & 84 & 7.21
\end{tabular}
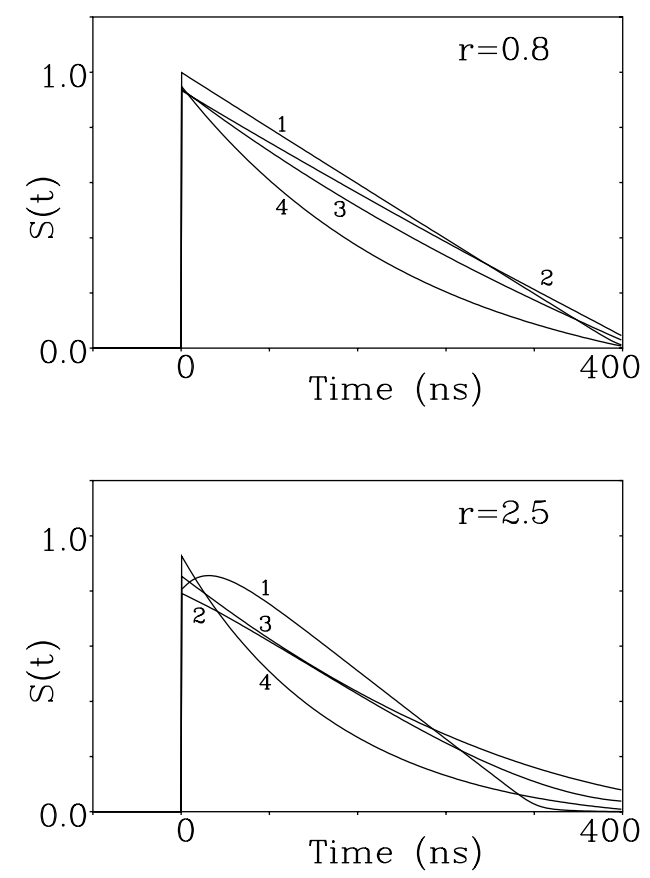

Fig. 13. Signal current vs. time for $r=0.8$ and $r=2.5$ for the same four simulation cases as in Fig. 11.

Case 3 differs from case 2 only in that $\mathcal{R}$ is increased to 5,000 .

Finally in case 4 we increased the relative (bulk) recombination rate $\mathcal{R}$ to the large value, $\sim 3 \times 10^{4}$, reported recently [22]. This value is outside the region of validity of the analytic approximations, Eq. (7.37). (See Fig. 8 and use $v_{-} / v_{+}=25,000$.) Table III sumarizes the simulation parameters. In all cases we used $\mu_{+}=0.2 \mathrm{~mm}^{2} / \mathrm{Vs}$.

Fig. 13 shows the signal current, $S(t)$, for $r=0.8$ and for $r=2.5$ for the four simulation cases. And $S_{i}$ and $S_{q}$ are shown as data points for eight values of $r$, including $r=0.8$ and $r=2.5$, on top of the analytic results of Fig. 11 . 
Simulation case 1 agrees well with the analytic calculations not only for the steady state backgrounds but also for the signal. The more realistic parameterization in simulation case 2 gives results not too different from the idealized case 1 . Deviations near $r=1$ were expected. The difference between case 2 simulations and the analytic calculations for $S_{i}$ at large $r$ are sensitive to our parameterization of $\mathrm{v}_{-}(E)$ at $E \gg V_{o} / a$ and cannot be trusted. Our case 2, 3 , and 4 parameterization gives a slow increase in $\mathrm{v}_{-}$with increasing $E$ while older data suggests that $\mathrm{v}_{-}$saturates at large $E$ (see Ref. [3,4]) and may actually decrease (see Ref. [9]) while newer data (Ref. [7,8]) does not cover this large $E$ range.

The initial current signal $S_{i}$ in simulation case 2 is better estimated by Eq. (9.22) shown as the middle curve near $r=1$ in the upper plot of Fig. 11. But Eq. (9.23) does an even better job of matching the simulated initial current signal $S_{i}$ as can be seen from the lowest curve near $r=1$. Both of these calculations use the electric field determined from the simple model of section 7 .

The integrated charge signal $S_{q}$ for cases 2,3 , and 4 at large $r$ is smaller than case 1 because the initial recombination in the region $z>z_{r}$ acts in the same way as large bulk recombination. See Eq. (9.21) and Eq. (8.1) and Eq. (8.3). Use of the exceptionally large value of $\mathcal{R}$ [22] on top of the parameterization in Eq. (10.1) and Eq. (5.2) (simulation case 4) depletes both some of the signal and some of the background charge. For $r<1$ depletion of the integrated charge signal, $S_{q}$, is significant but the initial current signal $S_{i}$ is actually enhanced due to the depletion of the background. For $r>1$ the integrated charge signal, $S_{q}$, agrees reasonably with the analytic approximation because the improved effects in the simulations largely cancel. But for the initial current signal, $S_{i}$, the depletion of the background gives a larger value for all $r>1$.

\section{Fluctuations in the Ionization Rate}

At a high intensity hadron collider such as the LHC the ionization deposited in the gaps of a liquid argon calorimeter is not uniform. There is an extensive literature, mostly in the form of collaboration notes, on these fluctuations. For $a=2 \mathrm{~mm}$ the drift time for electrons is about $400 \mathrm{~ns}$ and the drift time for the positive ions is of order $0.01 \mathrm{~s}$. At the LHC there will be of order 20 minimum bias interactions every $25 \mathrm{~ns}$. In the ATLAS detector the calorimeters are no farther than $5 \mathrm{~m}$ from the interaction point and the Moliere radius of EM showers in the parallel plate calorimeter of Fig. 1 would be about $21 \mathrm{~mm}$.

We identify four sources of fluctuations. 1) Particles do not strike the calorime- 
ter continuously but at every beam crossing. Because the particles of interest are relativistic there is no spread due to variations in transit time from the interaction point to the calorimeter. At the LHC beam crossings come every 25 ns. 2) At each beam crossing the number of minimum bias events varies about its average value of about 20. If the luminosity at each beam crossing is constant then the variation is Poisson distributed with mean and variance of 20. 3) For each minimum bias event the number of particles and each particle's energy and direction are random. Sophisticated Monte Carlo programs have been produced to simulate this random behavior. 4) Electromagnetic shower fluctuations at shower maximum are also simulated by Monte Carlo programs.

For $r<1$ the electron current from ionization produced in a single crossing is triangle shaped with an instantaneous rise to some initial current and a fall to zero in about $400 \mathrm{~ns}$. The positive ion current is similar but the fall to zero is much longer. Averaged over many crossings the electron current equals the positive ion current.

We will calculate fluctuations at $|\eta|=4.5$ in the area covered by a Moliere radius $R_{M}$ of $21 \mathrm{~mm}$ at a distance of $5 \mathrm{~m}$ from the interaction point. Such a size might be appropriate for a readout segment in the calorimeter.

If the initial current in each beam crossing were the same for all crossings (source number one above) then the fluctuation in the total current would be less than $0.5 \%$. So discrete beam crossings contribute a negligible amount to the total fluctuations.

We lump fluctuation sources two and three together and draw a result from Davis and Savard [23] that

$$
E_{T}^{r m s}=(0.367 \mathrm{GeV} / 0.1) \sqrt{A}+(0.10 \mathrm{GeV} / 0.1 \times 0.1) A
$$

where $A$ is the area in units of $d \eta \times d \phi$. (The 0.1 factors are for consistency with the units chosen in Ref. [23].) The parameterization is inspired by analogy to electronics noise where a portion is incoherent from channel to channel and a portion is coherent. In this case the coherent part (the part proportional to $A$ ) is due to low $p_{T}$ jets. So the parameterization is valid only up to area $A$ comparable to the size of a QCD jet. We use a small area $A=0.112$ so the fluctuations are dominated by the incoherent term. The parameterization applies to both electromagnetic and hadronic energy and is averaged over the response of the shaper circuit in the electronics which averages over several crossings. In this case the shaper time constant is such that the fluctuations are 1.5 times larger than for a single crossing. Because we want fluctuations in the EM calorimeter only we divide by $\sqrt{2}$ and to correct for the time constant of the shaper circuit we divide by 1.5. This gives the fluctuations in the energy (not $E_{T}$ ) deposited in the area $A$ as $E^{r m s}$ (EM only, 1 crossing) $=26.1 \mathrm{GeV}$. 


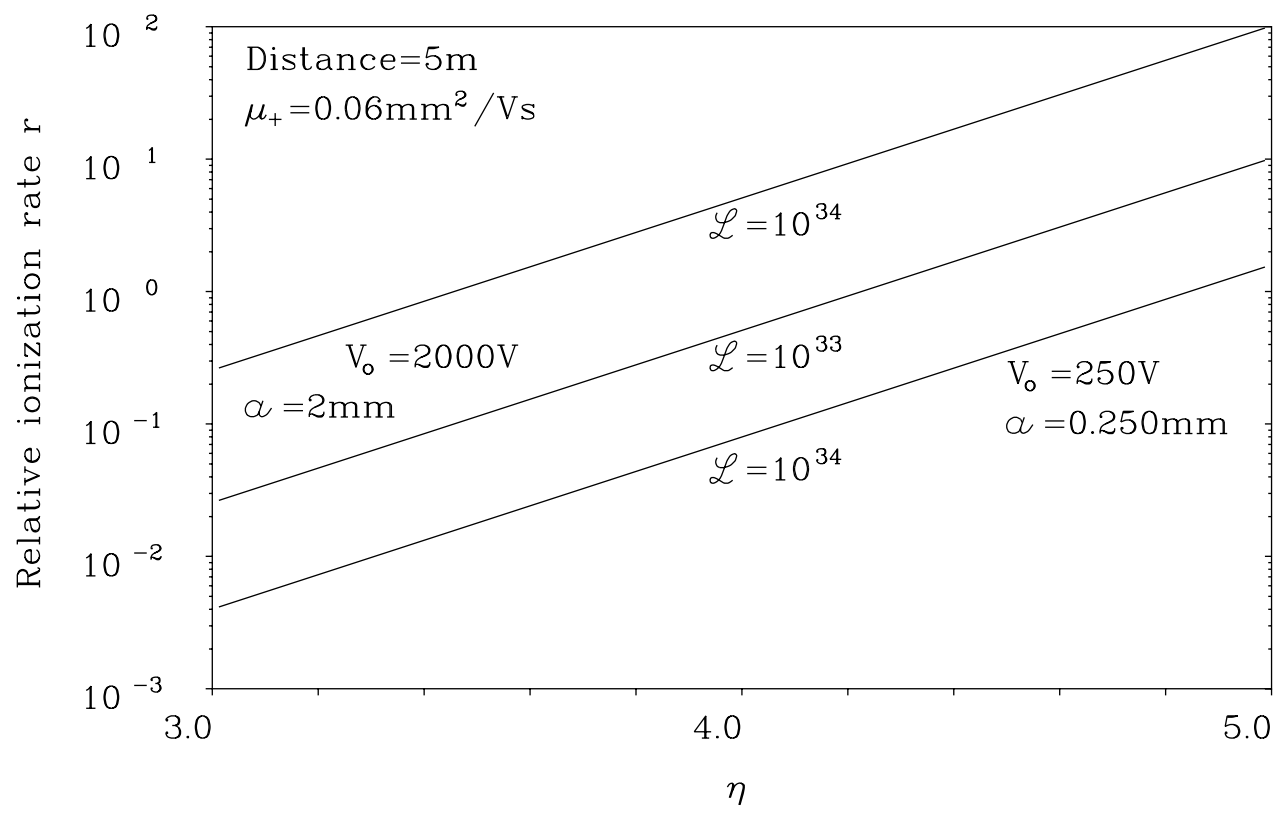

Fig. 14. For a forward calorimeter at a distance of $5 \mathrm{~m}$ from the interaction point (and assuming a positive ion mobility of $\mu_{+}=0.06 \mathrm{~mm}^{2} / \mathrm{Vs}$ ) the relative ionization rate $r$ at EM shower maximum changes with $\eta(\eta=\ln \tan \theta / 2$ where $\theta$ is the angle to the beam line) as shown for three cases: 1) canonical $2 \mathrm{~mm}$ gap with $2000 \mathrm{~V}$ potential at a luminosity of $10^{34} \mathrm{~cm}^{-2} \mathrm{sec}^{-1}, 2$ ) canonical $2 \mathrm{~mm}$ gap with 2000 $\mathrm{V}$ potential at a luminosity of $10^{33} \mathrm{~cm}^{-2} \mathrm{sec}^{-1}$, and 3) narrow gap of $a=0.250$ $\mathrm{mm}$ with $250 \mathrm{~V}$ potential (same average electric field) at a luminosity of $10^{34} \mathrm{~cm}^{-2}$ $\sec ^{-1}$.

The average EM energy per crossing deposited into this area is $\langle E\rangle=27.5$ $\mathrm{GeV}$. Shower fluctuations (source four) would normally be added in quadrature at this point but they are small by comparison and will be neglected.

The fluctuations averaged over many crossings are then found to be $14 \%$.

\section{Limits in the Operation of Liquid Argon Calorimeters}

To translate all of this to a specific situation we take the ATLAS detector at the LHC and we will make the assumption that the calorimeter will not yield acceptable operation above $r=1$. We will further assume that the calorimeter has gap width $a=2 \mathrm{~mm}$, potential across the gap of $V_{0}=2000 \mathrm{~V}$, that the face of the forward calorimeter is $5 \mathrm{~m}$ from the interaction point, and that $\mu_{+}=0.06 \mathrm{~mm}^{2} / \mathrm{Vs}$. This last assumption is very uncertain and makes the following conclusions rather tentative. (See section 4.) Fig. 14 shows the limits of operation of such a calorimeter at the LHC nominal luminosity of 
$10^{34} \mathrm{~cm}^{-2} \mathrm{sec}^{-1}$ and at the lower luminosity of $10^{33} \mathrm{~cm}^{-2} \mathrm{sec}^{-1}$.

At luminosities of $10^{33} \mathrm{~cm}^{-2} \sec ^{-1}$ the calorimeter beyond $|\eta|=4.2$ will be in the region $r>1$ and so, by our definition, will not work. At luminosities of $10^{34} \mathrm{~cm}^{-2} \mathrm{sec}^{-1}$ the calorimeter will cease to work above $|\eta|=3.45$. If the chosen value of $\mu_{+}$is low by a factor 2 , then the two limit lines in the figure will shift down by $\Delta \eta=0.2$. Raising $V_{o}$ to $4000 \mathrm{~V}$ (doubling the average electric field) will lower the lines in Fig. 14 by a factor 4 . This extends the operating range by $\Delta \eta=0.4$. But near $r=1$ the maximum electric field in the gap will be $4 \mathrm{KV} / \mathrm{mm}$ (see Fig. 6c) which may be difficult to achieve in this harsh environment. One could also decrease the gap width to 0.250 $\mathrm{mm}$ and the potential to $250 \mathrm{~V}$. This would lower the lines in Fig. 14 by a factor 64 increasing the range of operation of the calorimeter by $\Delta \eta=1$. . At the more likely value of $\mu_{+}=0.2 \mathrm{~mm}^{2} / \mathrm{Vs}$ a gap of $0.250 \mathrm{~mm}$ is sufficiently conservative to guarantee operation of a forward calorimeter at the highest LHC luminosities.

\section{Acknowledgements}

The author would like to thank Howard Gordon, Theodore Bowen, David Anderson, Michael Shupe, David Rahm, Paul Mockett, Hans Paar, Gerald Abrams, Geoffrey Forden, Andrew Milder, William Frisken, Jacques Colas, William Wenzel, and William Willis for helpful conversations and/or useful references.

This work was supported by Grant no. RFY9172 from the Texas National Research Laboratory Commission and Grant no. DE-FG02-85ER40213, Task B1 from the DOE.

\section{References}

[1] W.J. Willis and V. Radeka, Nucl. Instr. and Meth. 120 (1974) 221.

[2] Eido Shibamura et al., Nucl. Instr. and Meth. 131 (1975) 249.

[3] L.S. Miller, S. Howe, and W.E. Spear, Phys. Rev. 166 (1968) 871.

[4] K. Yoshino, U. Sowada, and W.F. Schmidt, Phys. Rev. A14 (1976) 438.

[5] V. Vuillemin et al., Nucl. Instrum. and Meth. A316 (1992) 71.

[6] A. Mozumder, IEEE Trans. Nucl. Science NS-26 (1979) 129.

[7] Wolfgang Walkowiak, Nucl. Instrum. and Meth. A449 (2000) 288. 
[8] A.M. Kalinin, Yu.K. Potrebenikov, A. Gonidec, and D. Schinzel, NA48 note 96-8, (1996); ATLAS Internal Note ATLAS-LARG-NO-058, CERN (1996).

[9] Morrel H. Cohen and J. Lekner, Phys. Rev. 158 (1967) 305.

[10] J. Lekner, Phys. Rev. 158 (1967) 130.

[11] Ref. [2] reports a null positive hole mobility in solid argon.

[12] W. Spear and P. LeComber in "Rare Gas Solids", vol. II, Ed. M.L. Klein and J.A. Venables, p. 1120 (1976). This reference reports a finite positive hole mobility in solid argon of $2 \mathrm{~mm}^{2} / \mathrm{Vs}$.

[13] T. H. Dey and T. J. Lewis, J. Phys. D 1 (1968) 1019.

[14] H. Ted Davis, Stuart A. Rice, and Lothar Meyer, J. Chem. Phys. 37 (1962) 947.

[15] Bob L. Henson, Phys. Rev. 135 (1964) A1002.

[16] Norman Gee, M. Antonio Floriano, Toshinori Wada, Sam S.-S. Huang, and Gordon R. Freeman, J. Appl. Phys. 57 (1985) 1097.

[17] R. L. Williams, Canadian J. Phys. 35 (1957) 134.

[18] Bret Halpern and Robert Gomer, J. Chem. Phys. 51 (1960) 1048.

[19] Kiyomitsu Arii and Werner F. Schmidt, IEEE Trans. Elect. Ins. EI-19 (1984) 16.

[20] J. Thomas and D.A. Imel, Phys. Rev. A36 (1987) 614; J. Thomas, D.A. Imel, and S. Biller, Phys. Rev. A38 (1988) 5793.

[21] Several authors have derived results similar to parts of the following. See for instance J. Colas, private communication and S. Palestini et al., Nucl. Instrum. and Meth. A421 (1999) 75. For the case of warm liquids see R.W. Kadel, private notes and W. Wenzel, private notes. For gas ionization chambers see J.W. Boag and T. Wilson, Brit. J. of App. Phys. 3, 222 (1952) and William C. Turner, LBNL-42180 (1998).

[22] K. Shinsaka et al., J. Chem. Phys. 88 (1988) 7529; K. Shinsaka and Y. Hatano, Nucl. Instrum. and Meth. A327 (1993) 7.

[23] R.A. Davis and P. Savard, ATLAS Internal Note, CAL-NO-084, 3/12/96. The equation is this author's fit to their Monte Carlo data. 